\title{
I. A Gentle Introduction to Structured Population Models: Three Worked Examples
}

\author{
J.A.J. Metz \& O. Diekmann
}

\section{Introduction}

In population models the basic unit is the individual. Therefore it is the task of the model builder to translate his/her knowledge about mechanisms on the individual level into models for the change in the number of such individuals. Generally, if one talks to an experimental ecologist (s)he has all kinds of alluring stories to tell about such mechanisms. However, as soon as it comes to writing down equations usually all that remains is but a handwaving reference when some mathematically convenient relationship between, say, death rate and population size is pulled out of the hat. The main reason for this unsatisfactory state of affairs probably is that applied mathematics seems to revolve around differential equation models which in the simplest case of ordinary differential equations necessarily start at a rather high phenomenological level. And biologists cannot but comply (but see e.g. MCKENDRICK (1926) for an early exception!). What clearly is needed, therefore, is a modelling methodology which in principle can accomodate any necessary amount of biological detail and yet is sufficiently near to the mainstream of applied mathematics that its tools can be brought to bear. This is the background to our efforts as set forward in these notes.

In this first section we shall give a very brief sketch of our modelling philosophy. If you find this sketch overly abstract we ask you to bear with us for a while: the examples in the next sections almost certainly will clarify the issue. A more detailed, and more technical, exposition of the basics of the modelling process may be found in chapter III. Chapter II proceeds with the general development of the mathematical toolkit that is needed to put the models thus derived to good use. This program is pushed on in chapters V and VI, while chapter IV concentrates on an alternative, slightly more phenomenological, modelling approach which can be very effective but applies only to a relatively special class of problems.

The basic concept in structured population modelling is that of an individual's state ( $i$-state; below the prefixes $i$ and $p$ - will be used to distinguish comparable concepts at the individual and population levels). Roughly speaking this amounts to a collection of variables which 1) at any one time fully determine the population dynamical properties of that individual, like rate of resource consumption or probability of dying or giving birth, and 2) such that their future values are fully determined by their present values and the intervening environmental history as encountered by that individual. Examples of potential $i$-state variables are satiation (of a predator), size, age or energy reserves.

Given a state representation of our individuals the population is conceived as a frequency distribution $n: \Omega \rightarrow \mathbb{R}^{+}$, where $\Omega$ denotes the set of possible $i$-states, the so-called $i$-state space. This distribution evolves over time due to physiological processes within the individuals, (local) removal of individuals from $\Omega$ due to deaths (or jumps to elsewhere in $\Omega$ ), and addition of individuals due to births (and returns from jumps). In this conception the frequency distribution $n$ fulfills the same role at the population level as the $i$-state fulfills at the individual level. Therefore we shall refer to it as the $p$-state. The question then is how to write down equations which do the right kind of bookkeeping, and given such equations how to extract information from them.

Before going on we should make clear that even if it is perfectly conceivable to construct stochastic population models of the structured kind, we shall confine ourselves in these notes to deterministic models only. This is not to say that an individual's behaviour is conceived as being completely fixed by its environment (in fact in almost all of our models stochastic effects at the individual level play an essential role), but only that there are sufficiently many individuals that any chance fluctuations deriving from individual stochasticity are completely ironed out.

Generally bookkeeping is easier for infinitesimally short time intervals as then the various processes contributing to population change tend to operate independently so that we can simply add their contributions. (On longer time scales we have to account for example for the fact that individuals that were eaten cannot give birth any longer). This explains the preponderance of differential equations as a modelling tool. But in the case of structured population 
models these cannot be ordinary differential equations as we need infinitely many numbers to characterize the $p$-state. In fact it turns out that they generally are (systems of) first order partial differential equations where the partial derivatives come from the smooth shift of the $p$-state due to continuous physiological processes. Apart from this the equations contain source and sink terms representing births and deaths respectively. An interesting point for mathematicians is that in general at least some of the source and sink terms are non-local, e.g. due to children being born from parents present elsewhere in the $i$-state space. To express this fact we shall frequently refer to the $p$ equations as functional partial differential equations.

It is when writing down expressions for the various terms in our bookkeeping operation that the second fundamental modelling concept comes into play, that of the "law of mass action". Adoption of this law amounts to saying that all the various individual contributions to for example source and sink terms, like births from different individuals or individual deaths can simply be added up. This summation then leads to the appearance of linear expressions like $\alpha_{0}(x) n(x)$ for a (local) disappearance rate due to random deaths (where $\alpha_{0}(x)$ is the probability per unit of time that any one individual in $i$-state $x$ dies), $\int \lambda(x, y) n(y) d y$ for a (local) birth rate (where $\lambda(x, y) d x$ denotes the (mean) rate at which one parent in $i$-state $y$ gives birth to children with $i$-states in an interval of size $d x$ around $x$ ), but also to bilinear expressions like $\int \alpha_{1}(x, y) m(y) d y \cdot n(x)$ for a (local) disappearance rate due to predation (where $\alpha_{1}(x, y)$ is the rate at which one predator in $i$-state $y$ searches for prey in $i$-state $x$ and $m(y) d y$ is the density of predators in an interval of $i$-states of size $d y$ around $y$ ), and also, through appropriate limit arguments, to the appearance of those partial derivatives referred to earlier.

Our first example below will indicate how starting from nothing but law of mass action considerations more complicated functional relationships may be derived by the use of time scale arguments. We want to stress, however, that it is only the basic derivation from law of mass action considerations which allows us to interpret the resulting relations in terms of individual behavioural characteristics.

Having derived population equations we can start studying them mathematically. One of the things we might do for example is simplify them by various kinds of limiting arguments. This may even lead to ordinary differential equations. But now the expressions occurring in these equations are not just phenomenological but can be interpreted in real, mechanistic terms. We may also go through the usual mathematical procedure of studying the large time behaviour of solutions, possibly with some numerical help in the later stages of the calculations, or we may immediately solve the equations numerically to obtain insight in the transient behaviour. The examples below and elsewhere in these notes will surely give you a taste of the various possible models and approaches subsumed under our general framework.

REMARK 1.1: Concerning the law of mass action: (i) The probabilistic nature of our models on the individual level intimately links the concepts of law of mass action and that of $i$-state. (A quantity like $\alpha_{0}(x)$ encountered above is nothing but a conditional probability per unit of time, where the condition is that the individual be in state $x$.) As far as we are concerned the detailed nature of this link still needs further clarification. Our present understanding of the matter is set forth in chapter III.

(ii) In our discussion of the $p$-state and the p-equation we were (and will remain) rather sloppy in that we constantly referred to population numbers whereas we meant densities i.e. numbers per unit of (spatial) area or volume. The reasons why densities and not numbers are paramount are a) our deterministic models dealing with smoothly varying quantities should rightly be interpreted as limits of stochastic models dealing with integers, the limit being taken by letting the area/volume under consideration grow large, and with it the number of individuals it contains, and b) only for $p$-equations dealing with densities are the coefficients in any bilinear expressions properties of the interacting individuals concerned: for example $\alpha_{1}(x, y)$ encountered above is the area/volume effectively searched per predator per unit of time; if we rephrase the $p$-equation in terms of numbers, by multiplying with the total area under consideration, at the place of $\alpha_{1}$ we get the fraction of the total area searched per predator per unit of time.

REMARK 1.2: Concerning the role of simulation methods. With the introduction of advanced simulation methods at the end of the sixties there also seemed to come a promise of bridging the gap between the mathematical models as studied by the theoretical community and the detailed mechanistic considerations as dealt with verbally by experimental ecologists. However, what actually happened is that a third breed of scientists emerged with their own preoccupations and linguistic pecularities. Naturally it is our hope that at least the language of structured population models will provide the necessary links. In this vision there are two important uses for the simulation approach: (i) at the level of the individual, where discrete stochastic simulation models closer represent an experimental biologists way of thinking; given such a simulation model it is usually fairly easy to re-express it in terms of an $i$-state and corresponding $i$-equations. And (ii) at the level of the population where simulation often is nothing but numerically solving the p-equations. 


\section{The invertebrate functional response}

\subsection{Introduction}

The very first models for predator prey interaction by VOLTERRA (1926) and LotKA (1925) were based on the law of mass action applied to whole predator and prey populations:

$$
\text { prey: } \quad \frac{d x}{d t}=r x-a p x \quad \text { predators: } \quad \frac{d p}{d t}=-c p+h a x p
$$

where $h$ is the conversion efficiency of prey into predator biomass. Population experiments by Gause (1934) soon led to a refutation: instead of the neutrally stable oscillations predicted, Gause found consistently that the oscillations immediately grew out of bounds, followed by extinction of the prey and subsequently the predator. His explanation was that there necessarily is a maximum to the rate at which a single predator can reproduce and/or ingest prey. Therefore at higher prey densities the amount of prey eaten and/or the number of offspring cannot be assumed to be simply proportional to prey density. A modification of the equations using various empirical formulae to take account of the 'satiation effect' indeed could generate the observed population developments (if also the assumption is made that a population went extinct when its density dropped below say one individual per culture vessel). The function relating prey density to number of prey eaten by a predator nowadays is called the functional response. Throughout this section we shall denote it as $F$.

EXERCISE 2.1.1: Write down the analog of equation (2.1.1) for a general functional response.

REMARK 2.1.2: The name functional response originates in the work of Solomon (1949) who rather loosely coined the phrase to describe the additional mortality at higher prey densities effected by predators, as opposed to the subsequent numerical response, i.e. population increase due to good feeding conditions, of those same predators.

The first detailed quantative experiments on predator feeding behaviour were done by IVLEV (1955). He found that for fish feeding on Daphnia the empirical formula

$$
F(x)=c\left(1-e^{-(a / c) x}\right)
$$

gave an exceedingly good fit to the number of prey eaten in his experiments. (Incidentally, the same formula was already used earlier by Gause to fit data on parasitoid reproduction). (2.1.2) is still commonly used by ecologists under the name of Ivlev functional response even if there really is not much to recommend it as the discussion below will make clear.

The first mechanistic models for the functional response were published in 1959 by HoLLING and by RASHEVSKY.

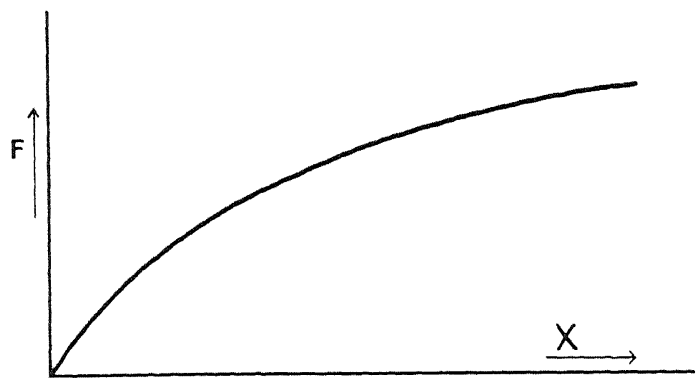

Fig. 2.1.1. Hollings "disk equation" functional response.

Holling started from a partitioning of the time budget of an individual predator. First of all he assumed that the number of prey caught per predator is proportional to prey density and the time spent in actual search. Then he noted that the time spent searching is less than the total amount of time allocated to foodgathering activities by the time needed to handle individual prey items. So, if $N$ denotes the number of prey caught during a foodgathering period, $T$ the duration of that period, $x$ the prey density, $a$ the search rate and $b$ handling time

$$
N=a x(T-b N) \text {, }
$$

and therefore 


$$
F(x)=\frac{N}{T}=\frac{a x}{1+a b x} .
$$

(See figure 2.1.1). In the ecological literature (2.1.4) is known as Holling's disk equation, after an experiment in which a blindfolded assistant searched for sandpaper disks laid out randomly on a table. The overwhelming advantage of (2.1.4) over purely empirical formulae like (2.1.2) is that all parameters in (2.1.4) have a direct empirical interpretation. This i) makes it possible to extend the model to other situations, like predators searching for a variety of prey items, and ii) makes the model and its extensions amenable to direct empirical testing. By now the model has spawned a whole industry of theoretical as well as empirical research.

Rashevsky's main inspiration were the experiments done by Ivlev. Therefore he took as his starting point a large predator snapping up small prey. He conjectured that the rate of search would be regulated mainly by the filling of the stomach. This he translated, apparently without any direct experimental evidence, into the assumptions that i) search rate decreases linearly with satiation, and ii) the rate at which satiation decreases due to digestion is precisely proportional to satiation. Combined with the usual law of mass action assumption this gave him for the satiation of the individual predator

$$
\frac{d s}{d t}=-\mu s+x a(1-s / c) \text {. }
$$

From this model he was able to explain Ivlev's formula (2.1.2) as the result of transient behaviour when very hungry fish were brought in contact with high food densities for a short time, in accordance with Ivlev's experimental protocol. The long term catch rate at constant food densities, however, is given by Holling's formula (2.1.4) with $b=(\mu c)^{-1}$. (See exercises 2.7.3 and 2.7.2).

In the ecological literature Rashevsky's work was largely ignored, and with it the role of satiation as an underlying $i$-state variable, until the publication in 1966 of another remarkable paper by Holling. In that paper Holling conceptually decomposed predatory behaviour into its component processes, measured all these processes in detail for a particular predator, the praying mantid Hierodula crassa, and finally reassembled the components in a relatively complicated computer simulation program. Independent experiments to test this model showed as good an agreement of observed and predicted catch as ever can be had in a model for a real organism.

Holling's mantid work was later refined, and extended to a number of other species, by various authors among whom FranSZ (1974) and SABELIS (1981, this volume). Yet the interest in satiation as an ecological variable remained mainly confined to the simulation literature. This probably is due to the seeming cumbersomeness of the resulting simulation models and certainly not to satiation being only a minor factor in determining functional responses. For as far as the data go generally just the opposite seems to be the case!

Below we shall derive a structured population version of Holling's "mantid" model due to METZ \& VAN BATENBURG (1984, $1985 \mathrm{a}, \mathrm{b})$. But before doing so we shall discuss how Holling's disk equation fits into the general framework laid out in section 1, as this allows us to introduce the necessary technical tools one at a time.

\subsection{Holling's disk equation 1: the underlying time scale argument, as exemplified by the finite state predator}

We shall start with a version of the model which can be formulated in terms of ordinary differential equations in order to ease the transition to the partial differential equations that will be needed eventually. To this end we assume that the predator can exist in only two states, 0 : searching and 1 : busy handling prey, leading to the population equations (p-equations)

$$
\begin{aligned}
& \frac{d x}{d t}=r x-a p_{0} x \\
& \frac{d p_{0}}{d t}=-a x p_{0}+\beta p_{1}+[\text { births }]-[\text { deaths }] \\
& \frac{d p_{1}}{d t}=a x p_{0}-\beta p_{1}+[\text { births }]-[\text { deaths }]
\end{aligned}
$$

Here we have intentionally left the birth and death terms unspecified as their specification becomes much easier after we have simplified our system of equations a bit.

To bring about this simplification we observe that generally prey densities are much higher than predator densities, and that moreover the rate of return from handling a prey, $\beta$, generally is of the same order of magnitude as the catch rate $a x$. This means that the exchange rates between states 0 and 1 is much faster than any changes in the overall predator density $p=p_{0}+p_{1}$ due to births or deaths. Moreover, the relative rate of decrease of prey density due to 
predation, $a p_{0}$, is small. We shall assume that $r$, the relative rate of change of $x$ due to births and (other) deaths, is of the same order of magnitude as $a p_{0}$. The consequence is that the relative proportions of predators in states 0 and 1 equilibrates much faster than either $x$ or $p$ changes.

We shall first deal with the short time scale. To this end we neglect the birth and death terms in (2.2.1b) and concentrate on the predation submodel per se:

$$
\frac{d p_{0}}{d t}=-a x p_{0}+\beta p_{1} \quad \frac{d p_{1}}{d t}=a x p_{0}-\beta p_{1}
$$

These equations necessarily satisfy the conservation condition $d\left(p_{0}+p_{1}\right) / d t=0$. Setting either $d p_{0} / d t$ or $d p_{1} / d t$ equal to zero and using the additional condition $p_{0}+p_{1}=p$ then gives the (pseudo) equilibrium values

$$
\tilde{p}_{0}=\frac{\beta p}{\beta+a x} \text {. }
$$

This equilibrium can easily be shown to be globally attractive in the linear manifold $p_{0}+p_{1}=p$. Using (2.2.3) we can calculate the functional response as

$$
F(x)=\frac{a x \tilde{p}_{0}}{P}=\frac{a x}{1+a b x},
$$

with $b=\beta^{-1}$.

Given the functional response it is easy to specify the birth and death terms for the predator population. First of all we have to account for biomass loss due to deaths and respiration, and secondly we have a biomass gain due to food intake. Together this gives (compare 2.1.1)

$$
\begin{aligned}
& \frac{d x}{d t}=r x-\frac{a x p}{1+a b x}, \\
& \frac{d p}{d t}=-c p+h \frac{a x p}{1+a b x} .
\end{aligned}
$$

(Note that we have artfully muddled the distinction between predator numbers and biomass. We simply have to do this if we want to neglect the size structure of the predator population!).

EXERCISE 2.2.1: Draw a picture of the linear manifold $p_{0}+p_{1}=p$ in the $\left(p_{0}, p_{1}\right)$-plane and sketch the vector field defined by (2.2.2). Also show analytically that the equilibrium (2.2.2) is stable within the manifold $p_{0}+p_{1}=p$. Hint: Eliminate $p_{1}$.

EXERCISE 2.2.2: Let $\pi_{i} \stackrel{d e f}{=} p_{i} / p$ so that $\pi_{0}+\pi_{1}=1$. Then $\pi_{i}, i=0,1$, also satisfies (2.2.2). And $F(x)=a x \tilde{\pi}_{0}$ where $\tilde{\pi}_{0}$ is the equilibrium value of $\pi_{0}$. Here $\pi_{0}$ and $\pi_{1}$ can be interpreted as the probabilities that an individual predator is in state 0 or 1 respectively. Seen from this vantage point the state process of the individual predator is a continuous time Markov chain with "differential generator" *

$$
B=\left[\begin{array}{rr}
-a x & \beta \\
a x & -\beta
\end{array}\right]
$$

where $b_{i j}$ is the transition rate from state $j$ to state $i$ and $b_{i i}$ is the rate at which state $i$ is left for any other state. Figure 2.2.1 shows a predator centered diagrammatic representation. What is the probability distribution of the handling time? What is the mean handling time? Hint: Consider a 'Gedanken-Experiment' in which at $t=0$ first the prey density is made infinite for a very short time and then all prey is removed. The probability that the predator still is busy handling prey at $t$ then is equal to the probability $\mathscr{F}(t)$ that the handling time is larger than $t$. The probability density of the handling time equals $-d \mathscr{F} / d t$.

- In contradistinction to the usual practice in probability theory we shall treat probability distributions as column vectors, as this fits better in the rest of our notation. 


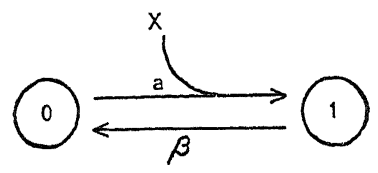

Fig. 2.2.1: Diagrammatic representation of the two state predator.

EXERCISE 2.2.3: Generalize the previous calculations to a predator eating two types of prey; to a predator eating $n$ types of prey.

EXERCISE 2.2.4: There is quite a lot of evidence that at least vertebrate predators start searching more efficiently for a prey when they encounter it sufficiently frequently, resulting in an overrepresentation of the commoner prey in the diet. The simplest finite state model that incorporates this effect is represented in fig. 2.2.2. Calculate the functional response for this model.

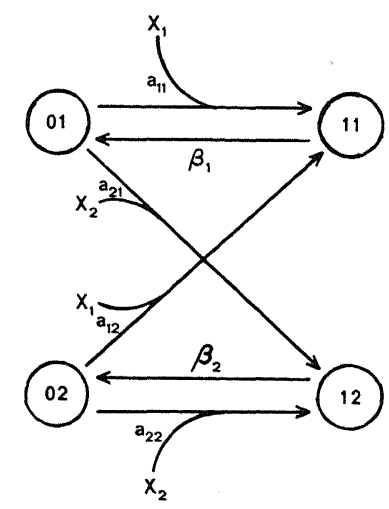

Fig. 2.2.2. The simplest 'switching' predator

REMARK 2.2.5: The problem of calculating functional responses for finite state predators is the same as that of calculating rate equations in enzyme kinetics. Enzyme kineticists have developped various efficient graphical techniques for deriving rate equations from more complicated reaction schemes like that in figure 2.2.2. See e.g. CORNISH-BOWDEN (1979) and WoNg (1975). The first rigorous exposition of the time scale argument may be found in HeINEKEN, TSUCHIYA \& ARIS (1967).

\subsection{Holling's disk equation 2: general handling times}

One not particularly biological feature implicit in the ordinary differential equation model is that prey handling time is assumed to be terminated randomly, i.e. the probability distribution of handling time is exponential (compare exercise 2.2.2), whereas in reality handling time distributions are usually strongly peaked around some fixed value. In itself the issumption of exponentially distributed handling times is no more artificial than the assumption that prey are born Ind die at random which underlies the ordinary differential equation form of the long term equation (2.2.5). However, there is the more serious question how $\beta$ can be estimated from observations made on the individual level. And the quantitative testing of any predictions derived from the model, and thereby the value of the model as a piece of scientific theory, hinges on our ability to make such estimates!

Below we shall indicate how in principle a structured population framework can be used to cope with general handling time distributions. In the end it turns out that it is only the mean handling time which counts.

REMARX 2.3.1: This result could also be arrived at in other ways, e.g. by using standard results from (Markov) 
renewal theory (Cox, 1962). The method chosen here, however, has the advantage that it can readily be extended to much more complicated models, e.g. incorporating satiation as well as handling time.

For didactical reasons we shall describe the basic ideas while confining ourselves to the case of a fixed handling time. General handling time distributions will be dealt with in the exercises at the end.

Let at any time $t$ the number of searching predators be $P_{0}(t)$ and the number of predators which are handling prey $P_{1}(t)$. (The reason for the capital letters will become clear below). However, predators busy handling prey differ in how long ago they encountered their prey, and this difference affects their future encounter rate by determining when they will restart searching. Therefore we assign to each busy predator the time $\tau, 0 \leqslant \tau<b$, elapsed since its last prey encounter (imagine a stopwatch which starts running at the moment our predator meets a prey), and introduce a function $p_{1}(t, \tau)$ such that the number of predators with internal clock time between $\alpha$ and $\beta$ is given by $\int_{\alpha}^{\beta} p_{1}(t, \tau) d \tau$.
Consequently

$$
P_{1}(t)=\int_{0}^{b} p_{1}(t, \tau) d \tau
$$

We shall refer to $p_{1}$ as the $\tau$-distribution, or, when we feel fussy, the $\tau$-density.

Clearly

$$
p_{1}(t, \tau)=p_{1}(t-\Delta, \tau-\Delta)
$$

whenever $\Delta<\tau<b$, and in particular

$$
p_{1}(t, b)=p_{1}(t-b, 0)
$$

A good way to visualize these relations is to imagine a heap of sand being transported by a conveyor belt (see fig. 2.3.1). In this image (2.3.3), for example, relates the amount of sand leaving the belt per unit of time to the amount put on it at the other end some while ago.

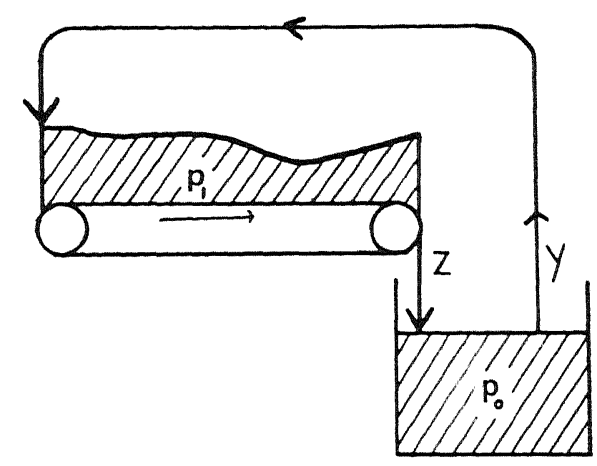

Fig. 2.3.1: The conveyor belt analogy for the structured population type formulation of Holling's disk model with fixed handling time.

The next step is to use (2.3.2) to derive a differential equation for $p_{1}$. Taking the limit $\Delta \downarrow 0$ in

$$
\Delta^{-1}\left[p_{1}(t, \tau)-p_{1}(t-\Delta, \tau)\right]=\Delta^{-1}\left[p_{1}(t-\Delta, \tau-\Delta)-p_{1}(t-\Delta, \tau)\right]
$$

we obtain the partial differential equation

$$
\frac{\partial p_{1}}{\partial t}(t, \tau)=-\frac{\partial p_{1}}{\partial \tau}(t, \tau)
$$

as a description of the way in which $p_{1}$ changes due to the movement of the belt (the running of the stopwatch) during infinitesimally short time intervals, for all positions $\tau>0$. To complete the description we have to specify how many predators step on the belt per unit of time. As during one time unit exactly one unit of belt length becomes available at its left hand end, $p_{1}(t, 0)$ precisely equals this "arrival rate". To derive this more formally let the arrival rate be denoted by $y$. Then

$$
\int_{0}^{\Delta} p_{1}(t, \tau) d \tau=\int_{t-\Delta}^{t} y(s) d s
$$


or after dividing by $\Delta$ and letting $\Delta$ go to zero

$$
p_{1}(t, 0)=y(t) .
$$

The usual law of mass action consideration lets us calculate $y$ as

$$
y(t)=a x P_{0}(t) .
$$

A relation like $(2.3 .4 \mathrm{~b})$ is called a boundary condition as it tells us something about the behaviour of $p_{1}$ at the boundary of its domain. 'Together (2.3.4b) and (2.3.4c) are also known as a side condition as in combination they amount to a restriction on the class of possible $p$-states $\left(P_{0}, p_{1}\right)$ at any one time.

The companion equation for $P_{0}$ is found from law of mass action considerations to be

$$
\frac{d P_{0}}{d t}=-a x P_{0}+z
$$

where $z$ is the rate at which predators leave the belt at $\tau=b$. By repeating the reasoning which led to (2.3.4b) we get

$$
z(t)=p_{1}(t, b) .
$$

Equation (2.3.4) forms a dynamical mathematical formulation of our simple model. This description moreover is such that if we provide an initial condition $P_{0}(0)=\Psi_{0}, p_{1}(0, \tau)=\psi_{1}(\tau)$ such that $\Psi_{0}+\int_{0}^{b} \psi_{1}(\tau) d \tau=P$ then $P_{0}(t)+\int_{0}^{b} p_{1}(t, \tau) d \tau=P$ for all $t$.

EXERCISE 2.3.2: Verify the last statement. Hint: Differentiate relation (2.3.1) for $t$, and substitute (2.3.4a) to arrive at $d P_{1} / d t=y-z$.

At equilibrium all derivatives with respect to time vanish. From (2.3.4a) we then find that the equilibrium value of $p_{1}, \hat{p}_{1}$, is constant, i.e. independent of $\tau$. Both $(2.3 .4 \mathrm{~b})$ with $(2.3 .4 \mathrm{c})$, and $(2.3 .4 \mathrm{~d})$ with $(2.3 .4 \mathrm{e})$ then lead to the conclusion that $\hat{p}_{1}=a x \hat{P}_{0}$. In combination with the conservation condition $b \hat{p}_{1}+\hat{P}_{0}=P$ this gives

$$
\hat{P}_{0}=\frac{P}{1+a b x}
$$

(Compare (2.2.3)), and therefore

$$
F(x)=\frac{a x \hat{P}_{0}}{P}=\frac{a x}{1+a b x}
$$

as before.

REMARK 2.3.3: The fact that we could either use (2.3.4b) and (2.3.4c) or (2.3.4d) and (2.3.4e) to arrive at $\hat{p}_{1}=a x \hat{P}_{0}$ shows that the various components of the equilibrium equation, derived from setting the time derivatives equal to zero, are not independent. It is therefore that we have to add the condition $b \hat{p}_{1}+\hat{P}_{0}=P$. All this is a direct consequence of the conservation of predator number inherent in (2.3.4). We already encountered this phenomenon in a more obvious fashion in the finite state predator. Exercise 2.2 .1 provides a geometrical illustration.

The mathematical justification for using the equilibrium catch rate (2.3.6) as a component of our model for the long term development of predator and prey populations hinges on the stability of the equilibrium (2.3.5). This we il not prove here. But after you have read the following chapters you should be quite able to repair this omission urself.

The exercises below put our predation (sub)model in some different, possibly illuminating, perspectives. They also :end the previous calculations to general handling time distributions.

EXERCISE 2.3.4: In a cooking timer you set the time needed to bring the cooking of a dish to completion, and then the timer starts to run backwards. We can just as well use this analogy instead of that of the stopwatch as a basis for our $i$-state description. Let $\sigma=b-\tau$ be the state of the cooking timer and let $q$ be the $\sigma$-density, then

$$
\frac{\partial q}{\partial t}(t, \sigma)=\frac{\partial q}{\partial \sigma}(t, \sigma)
$$

Derive this equation from first principles and also from (2.3.4) using the transformation rule 


$$
q(t, \sigma)=p_{1}(t, b-\sigma)
$$

Also draw the analogue of figure 2.3.1. What is the analogue of equation (2.3.4b)?

EXERCISE 2.3.5: Complete the partial p-equations derived in the previous exercise. What is the equilibrium? Calculate the functional response.

EXRRCISE 2.3.6: To give (2.3.6a) a direct biological interpretation consider a predator which only searches on an empty gut. Gobbling a prey brings its gut content, or satiation $s$, to $w$. Prey is digested at a constant rate $v$. So $b=w / v$. Write down population equations on the basis of the $i$-state variable $s$. Call the $s$-density $u$. As a check of your results also calculate the functional response from these equations. Finally rescale satiation by setting $\sigma=s / \nu$, and rederive the $p$-equation from the previous two exercises, using the substitution

$$
q(t, \sigma)=v u(t, v \sigma),
$$

where the factor $v$ derives from the fact that the $\sigma$-density $q$ has to accomodate as many predators on a length of conveyor belt $b=w / \nu$, as the $s$-density $u$ has to on a length $w$.

EXERCISE 2.3.7: Now consider the situation in which some prey are bigger than others, leading to a probability distribution of handling times with density $f$. Try to convince yourself that instead of (2.3.6a) we now get

$$
\frac{\partial q}{\partial t}(t, \sigma)=\frac{\partial q}{\partial \sigma}(t, \sigma)+y(t) f(\sigma) .
$$

First assume that there is no maximum to the possible handling times so that $q$ lives on the whole positive half axis. Complete the $p$-equations on this assumption and calculate the functional response.

Hint: Let the so called survivor function of the handling times be

$$
\mathscr{F}(\sigma)=\int_{\sigma}^{\infty} f(\rho) d \rho .
$$

Use partial integration to show that

$$
\int_{0}^{\infty} \mathscr{F}(\sigma) d \sigma=\int_{0}^{\infty} \sigma f(\sigma) d \sigma,
$$

the mean handling time.

EXRRCISE 2.3.8: Consider the same setting as the previous exercise, but now assume that there is a maximum $\sigma_{m}$ to the possible handling times, i.e. $f(\sigma)=0$ for $\sigma>\sigma_{m}$. It then seems expedient to restrict the domain of $q$ to the reachable set of $\sigma$ values, just as was done in exercise 2.3.4. In that case we have to add the boundary condition

$$
q\left(t, \sigma_{m}\right)=0 \text {. }
$$

Why? Check that at equilibrium (2.3.12) is automatically satisfied. Can you think of a reason why this should be the case? Hint: Look at remark 2.3.3.

EXERCISE 2.3.9: The variable handling time problem can also be set in a stopwatch as opposed to a cooking timer perspective. Use the relation

$$
p_{1}(t, \tau)=\frac{\mathscr{F}(\tau)}{\mathscr{F}(\tau-\Delta)} p_{1}(t-\Delta, \tau-\Delta)
$$

where $\mathscr{F}(\tau) / \mathscr{F}(\tau-\Delta)$ is the probability that a handling time will be longer than $\tau$ conditionally on it being longer than $\tau-\Delta$, to derive

$$
\frac{\partial p_{1}}{\partial t}(t, \tau)=-\frac{\partial p_{1}}{\partial \tau}(t, \tau)-\mu(\tau) p_{1}(t, \tau)
$$

with

$$
\mu(\tau)=-d \log \mathscr{F}(\tau) / d \tau=f(\tau) / \mathscr{F}(\tau)
$$

Complete the $p$-equation and calculate the functional response.

* EXERCISE 2.3.10 (for delay equation fans): Use (2.3.4e), (2.3.4b) with (2.3.4c) and (2.3.3) to reformulate (2.3.4d) as a linear autonomous retarded functional differential equation 


$$
\frac{d P_{0}}{d t}=-a x P_{0}(t)+a x P_{0}(t-b)
$$

Show that $\lambda=0$ is a root of the characteristic equation

$$
\lambda=a x\left(e^{-b \lambda}-1\right)
$$

and that all the other roots lie in the left half plane. Use this to prove that the solution to (2.3.4) converges exponentially to its equilibrium.

\subsection{The general invertebrate predator 1: the basic biology}

After the excursion into handling time based models we are now ready to consider the effect of satiation. Figure 2.4.1 shows a representation of the prey catching process broken down into its main components according to HoLLING (1966). The rectangular boxes correspond to the various directly observable activities of a generalized invertebrate predator, with between parentheses a reference to the form this activity takes in a particular predator, the praying mantid, which Holling studied in great detail. The duration and/or success of each of these activities may be influenced by the predator's satiation.

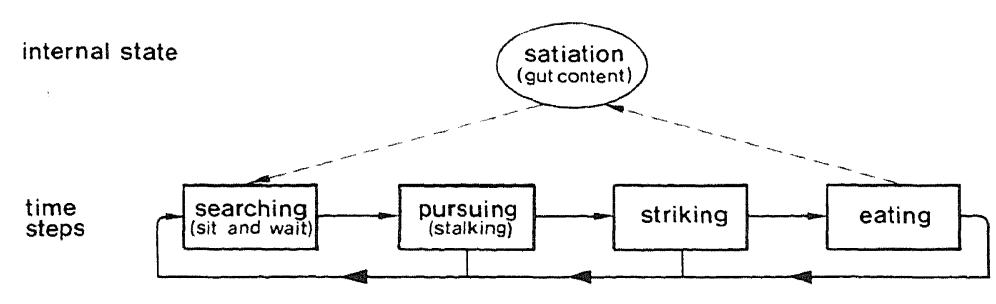

Fig. 2.4.1: Decomposition of the prey catching process according to Holling.

We shall defer to chapter III (sections 1, 5.1, appendix $B_{1}$, and exercises) the presentation of a complex model which takes into account both satiation and the durations of pursuit and eating. Often, however, these durations are so short that they can safely be neglected. In that case satiation remains as the only component of the $i$-state. Experiments (HolliNG (1966); another reference is SABELIS (1981)) suggest that, for invertebrates at least, satiation can effectively be considered a one-dimensional quantity, to be equated here naively with gut content, decreasing exponentially between meals and increasing in the course of a meal. If we neglect eating duration, we have to assume that the capture of a prey leads to an instantaneous upward jump of the satiation (compare exercise 2.3.6). The size of this jump will equal the prey weight $w$ if a prey is eaten completely (provided we measure satiation and prey weight in the same units) but it may be smaller when satiation is very high (see below). In between the meals the satiation dynamics is given by

$$
\frac{d s}{d t}=f(s)=-a s,
$$

where $a$ is the rate constant of digestion.

REMARK 2.4.1: The implicit assumption that prey weight has but one value can easily be relaxed. See METZ \& VAN BATENBURG (1985a).

The essential reason for introducing the $i$-state variable satiation is that the search rate is satiation dependent, or, in the terminology of the law of mass action, the rate constant of prey capture depends on it. We shall denote this rate constant as $g$. The rate at which an individual predator captures prey then is $x g(s)$

INTERLUDE 2.4.2: Determining $g$ from experimental data, the mantid case.

The rate constant of prey encounter during search is determined by the form and the size of the search field and the velocity 
distributions of predator and prey. In the case of a 'sit and wait' predator like the mantid, the predator's velocity is zero. Therefore the rate constant of prey encounter equals the mean prey velocity times the mean width of the search field (compare fig. 2.4.2). Holling found that this width decreases linearly with satiation until it becomes zero. To get the rate constant of prey capture we still have to multiply with pursuit and strike success. Strike success in the mantid is constant. Pursuit success can be calculated from the observation that prey escape during pursuit at a constant rate, that pursuit velocity is constant and that the distance that has to be bridged for a successful pursuit equals the distance at first sighting minus the distance bridged by the strike. The resulting formula for the satiation dependent rate constant of prey capture is

$$
g(s)=g_{0}(s) g_{1}(s) g_{2}(s)
$$

where $g_{0}(s)$ is the rate constant of prey encounter, $g_{1}(s)$ pursuit success and $g_{2}(s)$ strike success, and

$$
g_{0}(s)=b(1-s / c)^{+}, g_{1}(s)=\exp \left[-\beta(1-s / \gamma)^{+}\right], g_{2}(s)=q
$$

where the ${ }^{+}$indicates that a negative quantity should be replaced by zero, and where

$c$ is the satiation threshold, i.e. the value of $s$ at which the width of the search field becomes zero,

$b$ is the (mean) width of the search field times the (mean) prey velocity,

$\gamma=c\left(R_{m}-R_{s}\right) / R_{m}$, with $R_{m}$ the search radius at zero satiation and $R_{s}$ the distance bridged by a strike,

$\beta=\mu\left(R_{m}-R_{s}\right) / v$, with $\mu$ the escape rate of the prey and $v$ the pursuit velocity, and

$q$ is strike success.

(In the formula for $g_{1}$ we made the simplifying assumption that the search field is circular. See METZ \& VAN BATENBURG (1985a) for the general case).

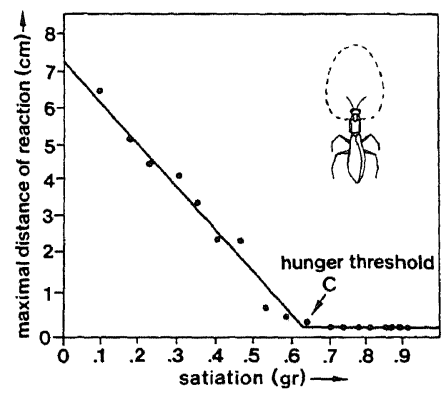

Fig. 2.4.2: Maximum distance of prey recognition as a function of satiation, and shape of the search field for the mantid Hierodula crassa. From Holuing (1966).

4

REMARK 2.4.3: A complication in Holling's mantid experiments was that the speed of the prey, house flies, appeared to decrease with prey density. This can be accounted for by interpreting $x$ as the effective prey density, i.e. the prey density multiplied by the speed reduction relative to the speed at zero density.
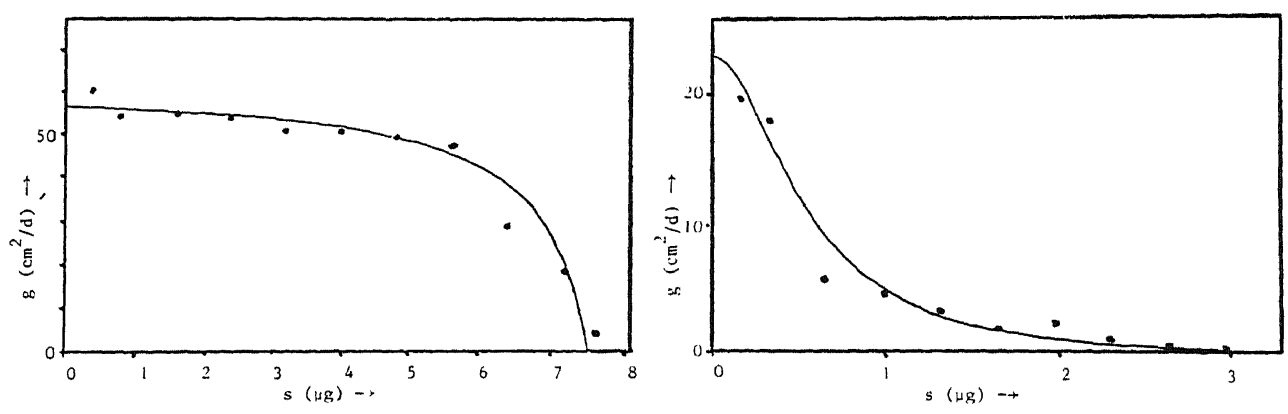

Fig. 2.4.3: Rate constant of prey capture as a function of satiation for the predatory mites Mesaseiulus occidentalis (left) and Phytoseiulus persimilis (right) feeding on eggs of Tetranychus urticae at $27^{\circ} \mathrm{C}$. Adapted from Sabelis (1981). 
We take $g$ to be a continuous, decreasing function of $s$ which vanishes for $s \geqslant c$ for some $c>0$. The previous interlude shows this to be the case for the mantid. As additional support for our assumption we refer to figure 2.4.3, adapted from SABELIS (1981) which shows $g$ for two different species of predatory mites feeding on spider mite eggs (see also the contributions of Sabelis to part B).

As a final point in the description of our predation (sub)model we still have to consider the biological detail which we rather brushed under the carpet at the start. Both Holling's mantid and Sabelis' mites still caught prey at satiations at which they were not able to eat all of its weight. When this happened they simply filled their gut to its maximum capacity $s_{m}$, and discarded the prey remains. (In Sabelis' case this effect in fact dominates the form of the functional response. In most of Holling's mantid experiments, including the final population experiments, prey size $w$ was in fact so small that $c+w<s_{m}$ ).

The functions $f$ and $g$, and the constants $w$ and $s_{m}$ totally specify our predation model. There remains the task of calculating the functional response from these ingredients. In contrast to the disk equation case there now is no straightforward simple argument which immediately yields the right answer, and we indeed have to put to work the whole machinery of deriving p-equations, showing that the dynamical system so defined allows a unique (globally) stable equilibrium state which can be calculated at least numerically, finally to obtain the functional response as the corresponding mean catch rate per predator.

\subsection{The general invertebrate predator 2: the population equation}

The $p$-state for our population of individually satiating predators corresponds to a density over the satiation axis, again denoted by $p$, so that $P(t,(u, v)):=\int_{u}^{v} P(t, s) d s$ equals the number of predators with satiation between $u$ and $v$ at time $t$, and (with some slight abuse of notation), for $\Omega=\left(0, s_{m}\right]$ the $i$-state space,

$$
\int_{\Omega} p(t, s) d s=P(t)
$$

where $P(t):=P(t, \Omega)$ is the total predator population size.

Deducing the $p$-equation is slightly more complicated than it was in our treatment of the disk equation as now the velocity of the $i$-state $f$ depends on $s$. Our conveyor belt from subsection 2.3 has become elastic: at some places it moves faster and at other places slower, and the differences in local velocities have to be accomodated by shrinking or stretching. In chapter III.3 we shall present a potpourri of systematic approaches to the general problem of setting up $p$-equations. Here we shall give but one, heuristic, derivation. For the devotees exercise 2.5.1 adds some more rigour.

To make things simple at the start we begin assuming that there are no prey present so that we may concentrate on the part of the $p$-equation representing the deterministic movement of individuals through $\Omega$ due to digestion. As we humans are better at reasoning with numbers or masses we shall first concentrate on $P\left(t,\left(s, s+\Delta_{s}\right)\right)$, the number of predators in a small satiation interval of length $\Delta_{s}$ beginning at $s$ (see fig. 2.5.1).

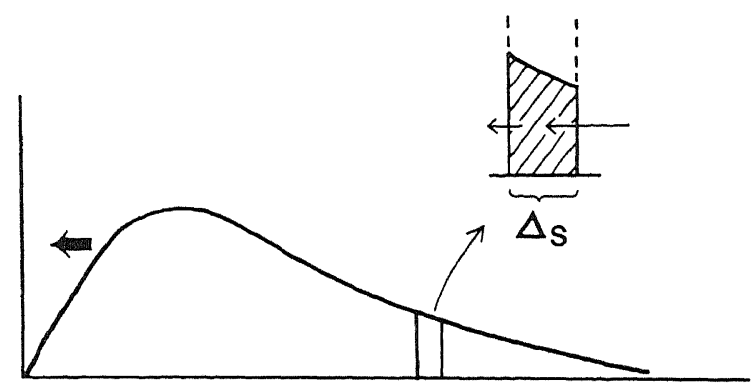

Fig. 2.5.1: The $p$-state of the predation (sub)model, and the subset of the total predator population in the satiation bracket $\left(s, s+\Delta_{s}\right)$. The horizontal arrows indicate movement of individuals due to digestion.

This number changes due to predators entering the satiation interval under consideration at its right hand boundary and predators leaving the interval at its left hand boundary. The two local flows equal the local speeds of the conveyor 
times the local predator densities. So, remembering that $f$ is negative,

$$
\frac{d P\left(t,\left(s, s+\Delta_{s}\right)\right)}{d t}=f(s) p(t, s)-f\left(s+\Delta_{s}\right) p\left(t, s+\Delta_{s}\right) .
$$

uming sufficient smoothness of $f$ and $p$ we may write*

$$
f\left(s+\Delta_{s}\right) p\left(t, s+\Delta_{s}\right)=f(s) p(t, s)+\frac{\partial f(s) p(t, s)}{\partial s} \Delta_{s}+o\left(\Delta_{s}\right)
$$

also

$$
P\left(t,\left(s, s+\Delta_{s}\right)\right)=p(t, s) \Delta_{s}+o\left(\Delta_{s}\right) .
$$

stituting this in (2.5.2), dividing by $\Delta_{s}$ and letting $\Delta_{s} \downarrow 0$ results in

$$
\frac{\partial}{\partial t} p(t, s)=-\frac{\partial}{\partial s}(f(s) p(t, s))
$$

$\mathrm{ch}$ is our sought equation.

ERCISE 2.5.1: Derive (2.5.2) by the following argument. For $\Delta_{t}$ sufficiently small the predators that by time $t+\Delta_{t}$ e left our satiation interval had at time $t$ satiation between $s$ and $s$ where $s$ is defined by $\sigma(t)=s$ with $\sigma$ satisfying $\checkmark d t=f(\sigma)$ with $\sigma\left(t+\Delta_{t}\right)=s$. So for small $\Delta_{t}$ we have $s=s_{-}^{-} f(s) \Delta_{t}^{-}+o\left(\Delta_{t}\right)$. Also calculate the number of dators that have entered. Use these quantities to calculate $\bar{P}\left(t+\Delta_{t},\left(s, s+\Delta_{s}\right)\right)$ and apply the usual limiting argunts.
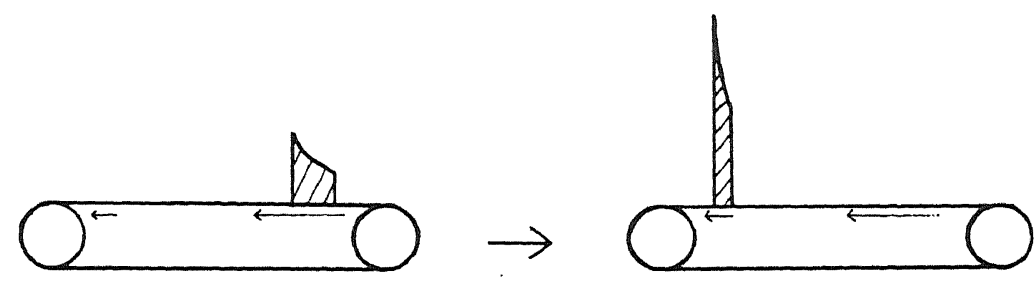

Fig. 2.5.2: The elastic conveyor belt at two subsequent times to illustrate how its contraction during its movement results in a higher stacking of the mass it carries.

Equation (2.5.3) can be rewritten as

$$
\frac{\partial}{\partial t} p(t, s)=-f(s) \frac{\partial}{\partial s} p(t, s)-p(t, s) \frac{d}{d s} f(s) .
$$

'hen $f$ is a constant only the first term remains (compare exercise (2.3.6). What then is the meaning of the second :rm? To understand this we return to our image of an elastic conveyor belt. (see fig. 2.5.2). At the right the belt Loves faster than at the left since digestion goes faster at higher satiation. Therefore the conveyor belt has to contract hile moving to the left. As a result the mass on it becomes stacked higher and higher as the length of conveyor belt vailable to it becomes shorter and shorter. It is this change in available length combined with the conservation of redator number which leads to the additional term $p(t, s) d f(s) / d s$.

iXERCISE 2.5.2: Assume that at $t=0$ a magician comes along and colours our predators in different shades of red, moothly depending on their current satiation. After this the magician vanishes into thin air in the customary manner - that our predators are doomed to stay the same colour for the rest of their ever more hungry lives. Let $y(t, s)$ be he colourshade of a predator which at time $t$ has satiation $s$. Derive a partial differential equation for $y$. (This probem also has some more useful counterparts in the modelling of our present magicwise rather impoverished world!)

- Jint: Use the approach from subsection 2.3.

- The notation $y=o(x)$ means that $y / x \rightarrow 0$ for either $x \rightarrow 0$ or $x \rightarrow \infty$, which of the two being clear from the context. In he present context $o\left(\Delta_{s}\right)$ means a term which is relatively negligable compared to $\frac{\partial f(s) p(t, s)}{\partial s} \Delta_{s}$. 
Now assume that we add highly toxic prey (at density $x$ ) so that the predators die immediately on catching a prey item. This changes (2.5.3) into

$$
\frac{\partial}{\partial t} p(t, s)=-\frac{\partial}{\partial s}(f(s) p(t, s))-x g(s) p(t, s)
$$

If the prey are not toxic a predator with satiation $s$ on catching a prey item still disappears at $s$, but only to "resurrect" higher up on the satiation axis, viz. at $s+w$. This means that to complete the bookkeeping at $s$ we have to add an arrival term representing the predators coming in from $s-w$. The final result is the functional partial differential equation

$$
\frac{\partial}{\partial t} p(t, s)=-\frac{\partial}{\partial s}(f(s) p(t, s))-x g(s) p(t, s)+x g(s-w) p(t, s-w) .
$$

(Functional, since the last term contains a so-called transformed argument making it non-local: the change of $p$ at $s$ depends on the value of $p$ at $s-w$.) Of course the meaning of the last term still has to be defined for $0<s \leqslant w$ as then $s-w \notin \Omega=\left(0, s_{m}\right]$. This will be taken care of in the following

CoNVENTION 2.5.3: If a transformed argument falls outside $\Omega$ we shall assume that the term in which it occurs equals zero.

Equation (2.5.5a) only tells us what happens inside $\Omega$. To this we have to add a boundary condition at $s_{m}$, the point of departure of the conveyor belt. In a small time interval of length $\Delta_{t}$ the number of predators whose eyes were bigger than their belly, and which therefore arrive at $s_{m}$, are

$$
\int_{s_{m}}^{s_{m}} x g(s) p(t, s) d s \Delta_{t}+o\left(\Delta_{t}\right) .
$$

These arrivals have to be accomodated on a stretch of conveyor belt of length $-f\left(s_{m}\right) \Delta_{t}+o\left(\Delta_{t}\right)$. The resulting side condition is

$$
-f\left(s_{m}\right) p\left(t, s_{m}\right)=\int_{s_{m}-w}^{s_{m}} x g(s) p(t, s) d s .
$$

REMARK 2.5.4: When $s_{m}>c+w(2.5 .5 \mathrm{~b})$ reduces to

$$
p\left(t, s_{m}\right)=0 \text {. }
$$

In that case after some initial period there will be no predators left with a satiation in $\left(c+w, s_{m}\right]$, i.e. $p(t, s)=0$ for $s>c+w$, and $t$ sufficiently large and therefore also, by continuity,

$$
p(t, c+w)=0 \text {. }
$$

If we also start without any predators in the upper satiation ranges we can just as well choose $\Omega=(0, c+w]$, using $\left(2.5 .5 b^{\prime}\right)$ as the boundary condition.

EXERCISE 2.5.5: Prove that the total number of predators

$$
P(t)=\int_{0}^{s_{m}} p(t, s) d s
$$

is constant by integrating (2.5.5a) from zero to $s_{m}$ and using (2.5.5b) to mop up the remains.

Hint: Use that $p$ should be integrable and that $f(s)=-a s$ as an indication that $\lim _{s \downarrow 0} p(t, s)=0$ (see also exercise 2.6.2). (Do not forget convention 2.5.3).

\subsection{The general invertebrate predator 3: calculating the functional response}

The equilibrium $p$-state of our predation model can be calculated from

$$
0=-\frac{d}{d s}(f(s) \hat{p}(s))-x g(s) \hat{p}(s)+x g(s-w) \hat{p}(s-w)
$$

together with the conservation condition 


$$
\int_{\Omega} \hat{p}(s) d s=P .
$$

(The side condition (2.5.5b) is satisfied automatically! See exercise 2.6.3.) A proof, using methods similar to those developed in chapter II, that the equilibrium is globally stable can be found in Heismans (1984).

To solve (2.6.1) one can start by taking $\tilde{p}(w)=\theta$, some arbitrary positive constant, and (numerically) integrating the ordinary differential equation (remember convention 2.5.3!) from $w$ down to 0 . Thereafter the known values of $\dot{p}$ on $(0, w]$ can be used to integrate from $w$ to $2 w$ and so on. Next one defines the auxiliary function

$$
\bar{p}(s)=\frac{\tilde{p}(s)}{\int_{\Omega} \tilde{p}(\sigma) d \sigma} .
$$

Since (2.6.1a) is linear it is also satisfied by $\bar{p}$. Finally we obtain $\hat{p}$ as $\hat{p}=P \bar{p}$.

Once $\bar{p}$ is calculated for various values of $x$ the functional response can be obtained from

$$
F(x)=x \int_{0}^{c} g(s) \bar{p}(s) d s .
$$

Figures 2.6.1 and 2.6.2 show some examples of $\bar{p}$ and $F$ calculated in this manner.
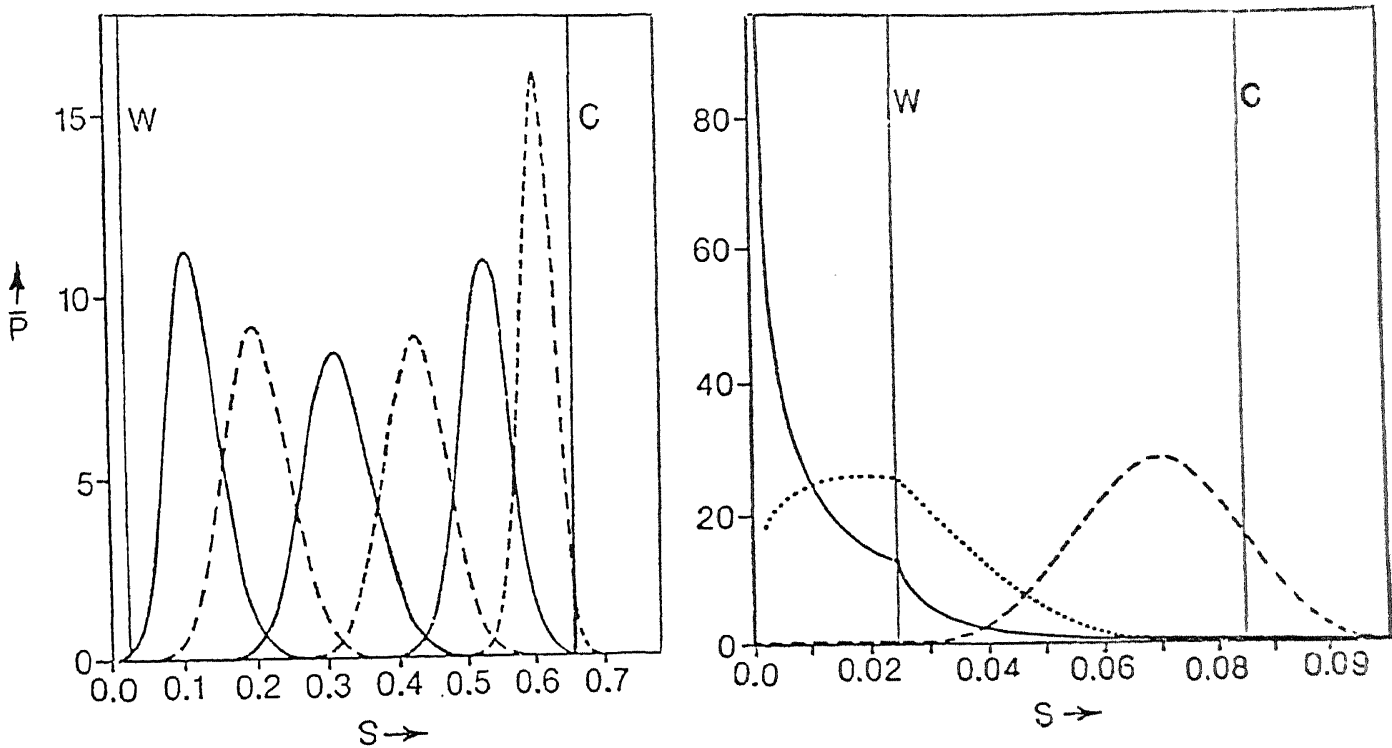

Fig. 2.6.1: The stationary satiation distribution for the mantids Hierodula crassa (left) and Mantis religiosa (right) for various values of the prey density. 

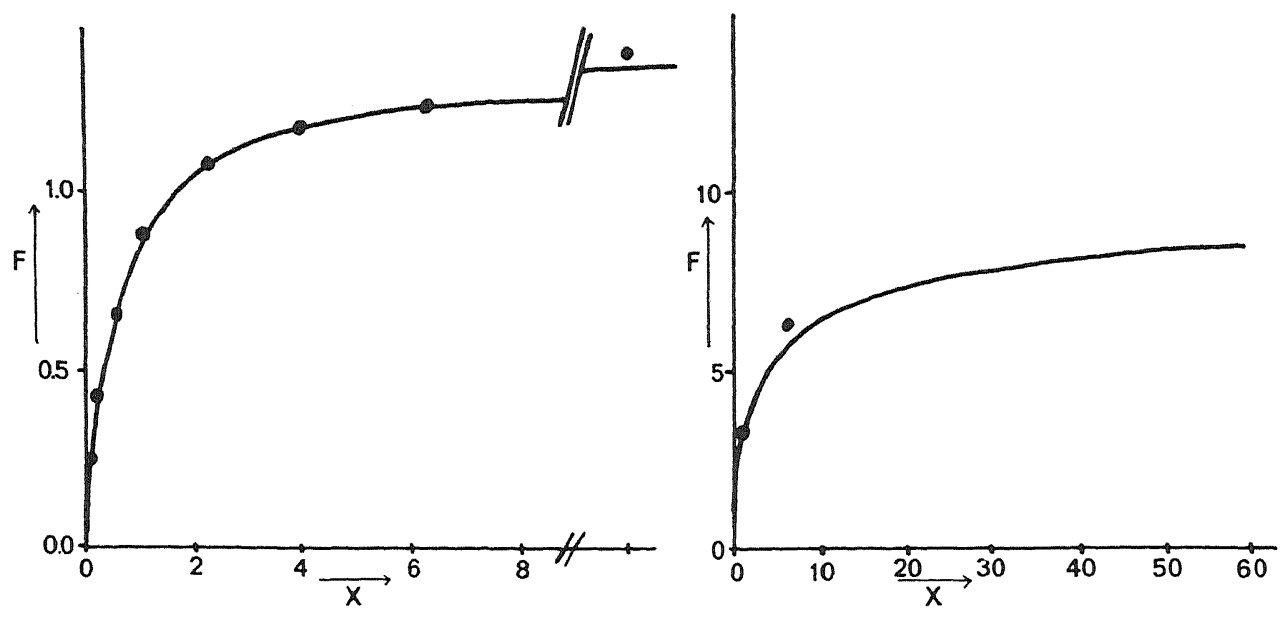

Fig. 2.6.2: The functional responses of the mantid Hierodula crassa and the mite Metaselulus occidentalis: exact calculation, - from approximation formula (2.7.5).

REMARK 2.6.1: Unless one takes great care numerical schemes for solving (2.6.1a) tend to be very unstable. (Good checks are whether positivity is conserved near $s=0$ and whether the side condition $(2.5 .5 \mathrm{~b})$ is approximately satisfied.) However, the straightforward scheme

$$
0 \approx f(n \Delta) \tilde{p}(n \Delta)-f((n+1) \Delta) \tilde{p}((n+1) \Delta)-g(n \Delta) \tilde{p}(n \Delta)+g((n-k) \Delta) \tilde{p}((n-k) \Delta)
$$

with $k=w / \Delta$ behaves very nicely. It not only guarantees the conservation of positivity but also ensures that an appropriately discretized version of the side condition (2.5.5b) is satisfied automatically. In SABELIS (1981, and part B) (2.6.4) is derived from a finite state predator model approximating our continuous one.

EXERCISE 2.6.2: Replace $g(s)$ by $g(0)$ to derive an approximation for $\tilde{p}$ on $(0, \epsilon], \epsilon<1$, assuming $\tilde{p}(\epsilon)$ to be known. What does $\hat{p}$ look like near $s=0$ ? And $f \hat{p}$ ?

EXERCISE 2.6.3: Prove that $\hat{p}$ automatically satisfies (2.5.5b). Also explain why this should be the case.

Hint: Integrate (2.6.1a) from 0 to $s_{m}$ and use the result from the previous exercise. A look at remark 2.3.3 also pays.

\subsection{The general invertebrate predator 4: The Rashevsky limit}

Although (2.6.1), (2.6.3) allow the numerical calculation of $F$ in specific cases, it is difficult to derive general properties from these equations. Moreover, we would like to have some relatively simple approximation formulae for $F$ for use in strategic models for the long term dynamics of prey and predator populations. We shall derive one such approximation here, assuming that the prey are very small but also very numerous. More extensive approximations may be found in METZ \& VAN BATENBURG (1985a; a proof of the essential correctness of the limit argument used below can be found in HeImans (1984).

For small but numerous prey it is the biomass that counts. Therefore we introduce as new variables the biomass density.

$$
\xi:=x w,
$$

and the biomass functional response

$$
\Phi(\xi):=w F \text {. }
$$


For small $w$ and sufficiently smooth $p$

$$
x g(s-w) p(t, s-w)=x g(s) p(t, s)-x w \frac{\partial}{\partial s}(g(s) p(t, s))+x \cdot o(w)
$$

and in the limit for $w \downarrow 0, x \uparrow \infty, \xi$ constant, equation (2.5.5a) reduces to

$$
\frac{\partial}{\partial t} p(t, s)=-\frac{\partial}{\partial s}((f(s)+\xi g(s)) p(t, s)),
$$

In (2.7.3) the jump contribution has been replaced by a contribution $\xi g(s)$ to the smooth movement of the satiation. In other words in the limit the originally particulate prey have been turned into some continuous broth which the predator just sucks in.

The $i$-state dynamics corresponding to (2.7.3) is described by the ordinary differential equation

$$
\frac{d s}{d t}=f(s)+\xi g(s)=-a s+\xi g(s),
$$

where $f$ accounts for digestion and $\xi g$ for ingestion (see figure 2.7.1). (2.7.4) is nothing but a slight generalization of Rashevsky's model described in subsection 2.1. A graphical argument immediately shows that for each $\xi>0$ (2.7.4) allows a unique globally attracting equilibrium $\hat{s}(\xi)$. For $(2.7 .3)$ this means that from everywhere our "conveyor belt" shrinks towards $\hat{s}$. So any initial distribution will contract in the course of time towards an infinitely peaked distribution, or delta "function", at $\hat{s}$.

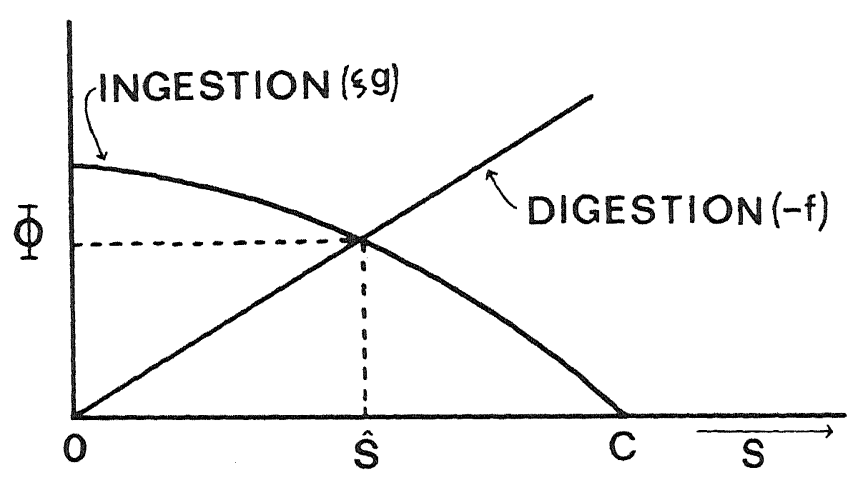

Fig. 2.7.1: The processes contributing to the satiation dynamics in the Rashevsky limit. Also indicated are the equilibrium satiation $\hat{s}$ and the value of the biomass functional response $\Phi$.

The biomass functional response for our limit model can be calculated as

$$
\Phi(\xi)=a \hat{s}(\xi)
$$

since

$$
\Phi(\xi)=\xi \int_{0}^{c} g(s) \delta(s-\hat{s}(\xi)) d s=\xi g(\hat{s}(\xi))
$$

and from the definition of $\hat{s}$

$$
\xi g(\hat{s}(\xi))=-f(\hat{s}(\xi))=a \hat{s}(\xi) .
$$

Figure 2.6.2 shows how (2.7.5) performs in two practical examples. (The large discrepancy between the numerically calculated functional response and the result of approximation (2.7.5) in the right graph is mainly due to the phenomenon of predators discarding half eaten prey at higher satiation. See SABELIS (1981) or METZ \& VAN BATENBURG (1985a)). The exercises provide some examples of $g$ for which $\Phi$ can be calculated explicitly. However, in practice a numerical solution of (2.7.6) is about as fast as using a complicated explicit formula. Any qualitative information, if needed, can relatively easily be extracted from (2.7.6). For example it is easily shown that $\Phi$ always increases with $\xi$ and that if $g$ is concave $\Phi$ is concave as well. 
EXERCISE 2.7.1: Prove the last statements. Hint: Differentiate (2.7.6) for $\xi$.

EXERCISE 2.7.2: Calculate $\Phi$ for $g(s)=\alpha(1-s / c)^{+}$(Rashevsky's choice for $g$ ) and for

$$
g(s)=\alpha\left(\frac{1-\beta s}{1-\gamma s}\right)^{+}, \beta>\gamma
$$

(the $g$ from figure 2.4.3 right).

EXERCISE 2.7.3: Rashevsky's explanation of Ivlev's findings. (In this exercise it pays to revert to the notation of subsection 2.1: $f(s)=-\mu s$ and $g(s)=a(1-s / c)^{+}$.) Ivlev's experiments i) were of short duration, ii) were done at high Daphnia densities, and iii) were done with (initially) starved fish. i) and ii) make that the digestion term in (2.7.4), or equivalently (2.1.5), can be neglected. Therefore the total amount of prey eaten equals the difference between the satiation at the start and at the end of the experiment. iii) means that the initial satiation was zero. Calculate the amount of prey eaten on the assumption that the duration of the experiment was one time unit. Compare the result with (2.1.2).

* EXercise 2.7.4: Let $r(t, s)$ be the solution to $d r / d t=h(r)$ with $h(r)=f(r)+\xi g(r)$ and $r(0, s)=s$. Then $r\left(t_{2}, r\left(t_{1}, s\right)\right)=r\left(t_{1}+t_{2}, s\right)$. Therefore $r(t, s)=r(t-\Delta, r(\Delta, s))=r(t-\Delta, s+\Delta h(s))+o(\Delta)$. Use this relation to show that

$$
\frac{\partial}{\partial t} r(t, s)=h(s) \frac{\partial}{\partial s} r(t, s)=h(r(t, s)) .
$$

Use this result to prove that the solution to $(2.7 .3)$ with $p(0, s)=\psi(s)$ equals

$$
p(t, s)=\psi(r(-t, s)) \frac{\partial r(-t, s)}{\partial s} .
$$

How should the two terms in the product be interpreted in the light of the discussion of the elastic conveyor belt in subsection 2.5 ?

\subsection{Concluding remarks and summary}

For pedagogical reasons we argued in this section mainly through worked examples. As a finish we shall go once more over the main steps, rephrasing them in general terms.

We started arguing that generally the time scales of the interaction of predator and prey populations on the one hand, and the predation process of individual predators on the other hand, differ to such an extent that we can model the latter while assuming that predator and prey densities (and age and/or size structures, if present) stay constant, and the former while assuming that the distribution of predators with respect to the relevant traits is in a pseudo equilibrium pertinent to the current prey density. This allowed us to concentrate on models for the predation process per se.

As a next step we gave a detailed analysis, following Holling (1966), of the various components of the predation process, culminating in the writing down of a linear functional partial differential equation describing how the $p$-state of the resulting predation model changes over time on an infinitesimal basis, as set forth in METz \& VAN BATENBURG $(1985 a, b)$. This equation generates a dynamical system which can be analyzed using the techniques from chapters II, IV and V. From this analysis it appears that in the linear manifold defined by the constancy of the total population size there exists a unique, globally attractive $p$-state, or stable $i$-state distribution. For various simple limiting cases this distribution can be calculated explicitly, but in general it will be necessary to revert to numerical calculations. After normalizing this distribution, so that its integral equals unity, it can be used to calculate the long term per capita predation rate as the average of the corresponding $i$-state specific rates.

In the following sections you will see various parts of this pattern of arguments repeated for total population models, in particular the extensive biological discussion, the deriving of functional partial differential equations, the calculation of stationary $i$-state distributions, and the use of these distributions to arrive at some biologically relevant end result.

** EXERCISE 2.8.1: The sketch of the argument above is slightly idealized, for we certainly did not prove everything we say. In fact there are still two open problems, both having to do with the structure of the time scale argument. In essence this is a singular perturbation argument using the predator prey ratio as the small parameter. Sketches of a more mathematical version, in an enzyme kinetical context, may be found in RUBINOV (1975) or MURRAY (1977). 
i) In many predator- prey models the predator and prey densities may go through huge fluctuations. For example, if in (2.2.5a) we add a term $-d x^{2}$ to represent self-limiting effects in the prey population, the system may show limit cycles which pass close to the axes. How does this affect the argument?

ii) As yet the general extension of the usual singular perturbation theory for ordinary differential equations to the class of functional partial differential equations describing the dynamics of structured populations still has to be made.

\section{Size dependent reproduction in ectothermic animals}

In our second example we turn to real population dynamics, using individual size as the main $i$-state variable.

Models for the growth of size structured populations were first derived in the late sixties by Sinko \& STREIFER (1967). Yet these models did not catch on as well as they should have done (otherwise there would have been no need to write these notes!) One probable reason is that the early workers did not fall back on specific physiological mechanisms but just left the equations in a fairly general form. This is very well if one wishes to prove general results. But for the interactive dealing with empirical data sufficiently specified parameter scarce families of models are almost a sine qua non.

Below we shall consider a specific model introduced by Koorsman \& MeTz (1984), and reformulated as a structured population model by Diekmann, METz, Koouman \& Heumans (1984). All functions involved will be specified immediately from first principles. The model was designed with simple ectothermic animals in mind. In our presentation we shall concentrate on the waterflea Daphnia magna as the experimental animal. More detail on the individual level as well as empirical evidence substantiating our assumptions can be found in Kooijman \& Metz (op. cit.). See the contribution by Kooijman in part B for a follow up.

\subsection{The dynamics of individuals}

We shall derive our $i$ equations by drawing up a balance of energy flows in combination with some dimensional considerations and a specific assumption about energy chanelling. We start our specification with a look at food intake, next we consider growth, reproduction and finally deaths. In the following $a$ will denote age, $l$ length and $x$ food density. We shall assume that during growth the animals just scale up in a selfsimilar way, and interpret $w:=l^{3}$ as weight (expressed in a suitable unit to avoid a constant of proportionality).

Our first assumption is that the ingestion of food by an individual of length $l$ at food density $x$, equals

$$
[\text { food intake }]=f(x) l^{2},
$$

where explicitly

$$
f(x)=\frac{\nu \xi x}{1+\xi x} .
$$

The basis for the assumption that ingestion is proportional to $l^{2}$ is that $i$ ) the maximum rate of food intake should equal the maximum digestion rate which scales with the surface area of the digestive apparatus, and ii) for filter feeders (and many other feeding types as well) the food intake at low food densities is proportional to the surface area of the feeding apparatus times the food density. The hyperbolic relation $(3.1 .1 \mathrm{~b})$ between food density and intake results from many micro models of the food catching process (e.g. HolLING (1959) and RashevsKy (1959); see subsection 2.1, and especially formula (2.1.4)). In the ecological literature it is known as the Holling (type II) functional response, in the microbiological literature as the Monod curve, and in the biochemical literature as Michaelis Menten uptake kinetics. Figure 3.1.1 shows some experimental evidence substantiating our choice of (3.1.1). 

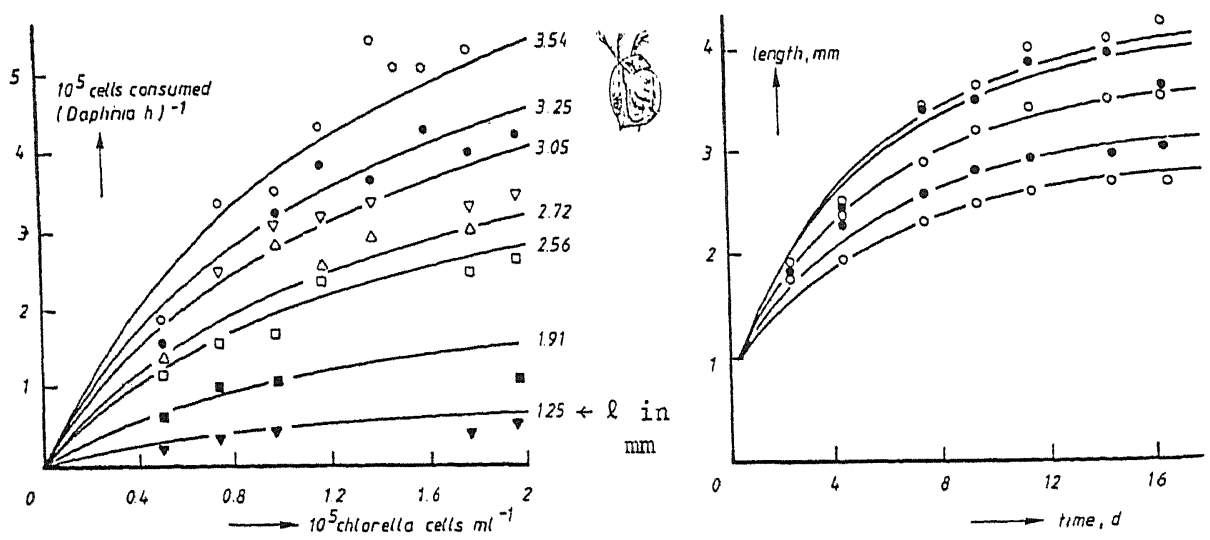

Fig. 3.1.1. Left: Feeding rate at $20^{\circ} \mathrm{C}$ of the waterflea Daphnia magna on the alga Chlorella as a function of food density $x$ and body length $l$. The fitted curves are given by $y=l^{2} f(x)$, with $f(x)=\nu \xi x /(1+\xi x)$ with $\nu=0.75 \times 10^{5}$ cells $/ \mathrm{h} . \mathrm{mm}^{2}, \xi=0.7 \times 10^{-5} \mathrm{ml} / \mathrm{cell}$. From KoOUuman \& MeTZ (1984). Data from MCMacoN \& RIGLER (1963), reproduced by WULFF (1980).

Right: length $/$ of Daphnia magna as a function of age $a$ for various food densities $x$. The fitted curves are given by $l=\exp (-\gamma a)\left(l_{b}-\underline{l}(x)\right)+\underline{l}(x)$, with $\underline{l}(x)=2.89,3.24,3.72,4.17,4.31 \mathrm{~mm}, l_{b}=0.8 \mathrm{~mm}$ and $\gamma=0.17 d^{-1}$.

To arrive at the individual growth equation we assume that a fraction $\kappa$ of the ingested food is chanelled to maintenance and growth, and a fraction $1-\kappa$ to reproduction. The rate at which maintenance needs energy (expressed in food units) is assumed to be $\zeta w$, where $w$ is the metabolizing tissue weight. What remains for growth is $\kappa f(x) w^{2 / 3}-\zeta w$, provided this quantity is positive. We assume that when it is negative animals stay of constant size (sea-anemones and flatworms do shrink during food scarcity, but more highly organized animals like Daphnids generally don't), the necessary maintenance energy being rechanelled from reproduction. Thus, remembering that $l^{2}=w^{2 / 3}$, we obtain a growth equation of so-called von Bertalanffy type (VON BERTALANFFY, 1934)

$$
\frac{d w}{d a}=\eta^{-1}\left(\kappa f(x) w^{2 / 3}-\zeta w\right)^{+},
$$

where $\eta$ is a conversion factor relating weight units to food units and $z^{+}:=\max (0, z)$.

Below we shall use length as our $i$-state variable in preference to weight, as it both leads to simpler equations and, at least for small aquatic animals, is easier to measure non- destructively. Transforming (3.1.2) to length (using $d l / d a=(d l / d w)(d w / d a))$ and defining

$$
g(x, l)=(3 \eta)^{-1}(\kappa f(x)-\zeta l)^{+}
$$

we find

$$
\frac{d l}{d a}=g(x, l) \text {. }
$$

For Daphnids, as for all animals that go through a regular reproductive cycle, birth length is constant to a very good approximation. Therefore we supplement $(3.1 .3 \mathrm{a}, \mathrm{b})$ with the initial condition

$$
l(0)=l_{b} .
$$

Figure 3.1.1 also shows some laboratory observations on the growth of Daphnia magna at constant food densities together with the fitted solutions to (3.1.3) as a further substantiation of our assumptions.

To calculate the individual reproductive rate we recall that a fraction $1-\kappa$ of the ingested food was assumed to be chanelled to reproduction. However, Daphnids (as most other invertebrates) only start reproducing when they have grown sufficiently large. (Presumably the "reproductive" energy surplus is invested in building up the reproductive apparatus and other pieces of body equipment without adding to overall growth; we certainly do not see an abrupt 
change in growth rate at the abrupt start of reproduction.) The length at which reproduction starts will be called $l_{J}$. Moreover we had introduced a(nother) modification of the energy chanelling rule as maintenance was assumed to take priority over reproduction when food densities drop so far that the animal stops growing. Taking stock we assume that the individual size specific birth rate $\lambda$ is given by

$$
\lambda(x, l)= \begin{cases}0 & \text { for } l_{b} \leqslant l<l_{J}, \\ \left(\omega w_{b}\right)^{-1}(1-\kappa) f(x) l^{2} & \text { for } l_{J} \leqslant l \leqslant l(x), \\ \left(\omega w_{b}\right)^{-1}\left(f(x) l^{2}-\zeta l^{3}\right) & \text { for } \max \left(l_{J}, l(x)\right) \leqslant l \leqslant \bar{l}(x),\end{cases}
$$

where $w_{b}=l_{b}^{3}$ and $\omega w_{b}$ is the amount of food needed to produce one young,

$$
\underline{l}(x):=\zeta^{-1} \kappa f(x)
$$

is the size at which growth just stops at the current food density, and

$$
\bar{l}(x):=\zeta^{-1} f(x)
$$

is the size for which at the current food density precisely all ingested food is needed for maintenance. We assume that animals for which $l>\bar{l}(x)$ die instantaneously, in order to be consistent with our earlier assumptions about energy chanelling and maintenance. (In part B Kooijman develops a model which also accounts for energy reserves).

Finally we have to specify the death rate. It appears that, apart from deaths from starvation when $\bar{l}(x)$ crosses $l$, the death rate is mainly determined by age and not by size, at least under laboratory conditions. This observation would entail the introduction of age as a second state variable (and we shall do so in III.1), but here we shall make the simplifying assumption of a constant death rate, denoted as $\mu$, while easing our conscience with the observation that under natural conditions deaths from old age are usually negligible compared with deaths due to other hazards such as predation.

Our description of the $i$-dynamics being completed we conclude this subsection with stressing once more that both the strong size dependence of reproduction observed in most ectothermic animals and the size dependence of resource use force us to take account of size as an $i$-state variable. Other aspects of the Daphnia life cycle, like the production of males and subsequently winter eggs (see fig. 11 in Kooijman in part B) as a reaction to deteriorating circumstances, we deliberately leave out of the model. (Likewise we do not consider the effect of temperature fluctuations.) This leaves us with $l$ as the only $i$-state variable with associated $i$-state space the interval

$$
\Omega=\left[l_{b}, l_{m}\right),
$$

where

$$
l_{m}=\zeta^{-1} \kappa \nu
$$

is the absolute upper bound to the individual length.

EXERCISE 3.1.1.: Calculate $l$ as a function of $a$ at constant food density.

EXERCISE 3.1.2.: Calculate the total number of offspring produced per individual during its lifetime at constant food density.

\subsection{Formulation of the population equations}

The $p$-state corresponds to a density over $\Omega$, to be denoted as $n$. So $\int_{a}^{b} n(t, l) d l$ is the number of animals with lengths between $a$ and $b$ at time $t$. (Or more precisely the number of animals per unit of volume; in the following we shall omit this qualification.) Using exactly the same reasoning as in section 2.5 we find that

$$
\frac{\partial}{\partial t} n(t, l)=-\frac{\partial}{\partial l}(g(x, l) n(t, l))-\mu n(t, l) \quad \text { for } l_{b}<l \leqslant \bar{l}(x),
$$

while, of course,

$$
n(t, l)=0 \text { for } l>\bar{l}(x) .
$$

At $l_{b}$ we have an influx of newborn individuals equal to the population birth rate $b(t)$. This influx should match the flux of individuals away from $l_{b}$, which equals $g\left(x, l_{b}\right) n\left(t, l_{b}\right)$, the product of the local individual "velocity" (i.e. the $i$ growth rate) and the local density (compare the derivation of (2.3.4b) and (2.5.5b)). The total number of births per 
unit of time equals the integral of $\lambda$ times $n$. Therefore $n$ should satisfy the side condition

$$
g\left(x, l_{b}\right) n\left(t, l_{b}\right)=b(t)
$$

with

$$
b(t)=\int_{\Omega} \lambda(x, l) n(t, l) d l .
$$

REMARK 3.2.1: Our model formulation contains one hidden assumption which is slightly embarassing from a biological point of view: the production of young is assumed to depend instantaneously on energy intake. In other words, an individual needs not accumulate the necessary energy $\omega w_{b}$. Thus we implicitly assume that all individuals at each time add some infinitesimal amount of young tissue to a communal pool from which, by some miracle, individual young are created. This assumption is commonly made in the literature of mathematical biology, but always implicitly. However in III.6.3 it will be shown that (3.2.1) can be justified as an approximation for the case when very many young of a very small weight are produced, each of which has only a small chance of surviving its early youth. Aldenberg's contribution in part B is bent on clarifying the confusion in the theory of biological production created by this hidden assumption.

\subsection{Constant environments}

If we wish to use (3.2.1) to arrive at conclusions about the possible behaviour of Daphnia populations we shall have to specify the dynamics of the food density $x$. The simplest assumption that we can make is that $x$ is constant. This will e.g. be approximately the case when the total population density is still very small and the food population (algae in the case of Daphnia) is at its own dynamical equilibrium. Anyhow, throughout this and the next subsection we assume $x$ to be constant and sufficiently large for $l(x)$ to be larger than $l_{J}$ (for otherwise no Daphnia would ever reproduce).

Generalizing from other models for density independent population growth one guesses that asymptotically (for $t \rightarrow \infty)$ the population will grow exponentially and that the length distribution will stabilize. The theory developed in chapters II, IV and V shows that this is indeed the case. Here we shall confine ourselves to calculating the asymptotic growth rate and the stable size distribution.

Mathematically our guess amounts to*

$$
n(t, l)=c e^{r t}(\psi(l)+o(1)) \text { for } t \rightarrow \infty,
$$

where $r$ is the (asymptotic) specific growth rate of the population, $\psi$ the stable length distribution and $c$ a constant depending on the initial condition. Neglecting the $o(1)$ term we insert (3.3.1) into the differential equation (3.2.1a) to find

$$
\frac{d}{d l}(g(x, l) \psi(l))=-(\mu+r) \psi(l)
$$

This equation should hold for $l_{b} \leqslant l \leqslant \bar{l}(x)$, as $\psi$ has to vanish for $\bar{l}(x)<l<l_{m}$. In order to simplify the notation we write

$$
g(x, l)=\gamma(\underline{l}(x)-l)^{+}, \quad \gamma:=\zeta /(3 \eta) .
$$

Solving (3.3.2) gives us the stable size distribution as

$$
\psi(l)= \begin{cases}\left.\psi\left(l_{b}\right)(\underline{\underline{l}(x)-l})^{\frac{r+\mu}{\gamma}-1}\right)^{\frac{l_{b}}{\gamma}-1} & \text { for } l_{b} \leqslant l<\underline{l}(x) \\ 0 & \text { for } \underline{l}(x)<l<l_{m},\end{cases}
$$

where $\psi\left(l_{b}\right)$ is still at our disposal as a free parameter and can be used to satisfy a normalization condition, such as the natural one

- $o$ (1) refers to a term which becomes arbitrarily small for sufficiently large $t$, i.e. o(l) $\rightarrow 0$ for $t \rightarrow \infty$. 


$$
\int_{\Omega} \psi(l) d l=1
$$

(see also exercise 3.3.4)

In (3.3.4) we have found an explicit expression for the stable size distribution, involving the still unknown population growth rate $r$. To calculate $r$ we substitute (3.3.1), neglecting the $o(1)$ term, into the side condition (3.2.1c,d) to arrive at the characteristic equation

$$
1=\pi_{x}(r),
$$

where by definition

$$
\pi_{x}(s)=\frac{1}{g\left(x, l_{b}\right)} \int_{l_{b}}^{\underline{l}(x)} \lambda(x, l)\left(\frac{\underline{l}(x)-l}{\underline{l}(x)-l_{b}}\right)^{\frac{s+\mu}{\gamma}-1} d l,
$$

and hence

$$
\pi_{x}(s)=\frac{(1-\kappa) f(x)}{\omega w_{b} \gamma\left(\underline{l}(x)-l_{b}\right)_{1}} \int_{L}^{l(x)} l^{2}\left[\frac{\underline{l}(x)-l}{\underline{l}(x)-l_{b}}\right)^{\frac{s+\mu}{\gamma}-1} d l
$$

(see exercise 3.3.3 below for an evaluation of the integral). The integral in (3.3.7) diverges for $s \leqslant-\mu$ and consequently $\pi_{x}$ is defined for $s>-\mu$ only (we shall only consider real arguments in accordance with the biological interpretation). Since $(l(x)-l) /\left(l(x)-l_{b}\right)<1$ for $l_{J} \leqslant l<l(x)$ the integrand in (3.3.7) strictly decreases with $s$. Therefore $\pi_{x}(s)$ strictly decreases too for $s>-\mu$. Moreover $\lim _{s \downarrow-\mu} \pi_{x}(s)=\infty$ and $\lim _{s \uparrow \infty} \pi_{x}(s)=0$. We conclude that (3.3.5) has a unique simple ${ }^{*}$ real solution $r$.

$r$ corresponds to what in the classical age-dependent theory is called the intrinsic rate of natural increase (compare subsection 3.4). The advantage of the mechanistic approach followed here is that it allows $r$ to be calculated as a function of the food density $x$, thereby making our theoretical developments amenable to experimental tests.

For practical applications equation (3.3.5) has to be solved numerically. However, many qualitative properties of $r$ can be derived without actually solving it. An example is provided by the extremely useful equivalence

$$
\pi_{x}(0) \geq 1 \Leftrightarrow r \geq 0 .
$$

(In the next subsection we shall derive a biological interpretation of the quantity $\pi_{x}(0)$.) It is also relatively easy to show that $r$ strictly increases with $x$, as is to be expected. Without loss of generality we may assume that $r>0$ for sufficiently large $x$ (biological generality: animals whose populations go extinct at any food density do not exist). Moreover $r \downarrow-\mu$ for $x \downarrow \underline{x}$ defined by $\underline{l}(x)=l_{J}$ (see exercise 3.3.2 below). We conclude that there exists a unique food density $x_{c}$ defined by

$$
\pi_{x_{\mathrm{r}}}(0)=1 \text {, }
$$

such that $-\mu<r<0$ for $x<x_{c}$ and $0<r<r_{m}$ for $x>x_{c}$, where $r_{m}$ is the solution to $\pi_{\infty}(r)=1$. So $x_{c}$ is the critical food density which the population needs to survive. For given values of the model parameters $\mu, \nu, \xi, \zeta, \eta, \omega, l_{b}$ and $l_{j}$ one can easily calculate $x_{c}$ numerically from the defining relation (3.3.9). So altogether we are able to give a rather neat and intuitively appealing characterisation of the dependence of the intrinsic rate of natural increase on the food density $x$.

EXERCISE 3.3.1: Prove (3.3.8).

EXERCISE 3.3.2: Prove that $r$ strictly increases with $x$, and that $r \downarrow-\mu$ for $x \downarrow \underline{x}$. Hint: use that $\pi_{x}(s)$ decreases with $x$, and that $\pi_{x}(s) \downarrow 0$ for $x \downarrow \underline{x}$, for all $s>-\mu$.

EXERCISE 3.3.3: Show that

$$
\pi_{x}(s)=\frac{(1-\kappa) f(x)}{\omega w_{b}}\left\{\frac{\underline{l}^{2}(x)}{\mu+s} A^{\frac{\mu+s}{\gamma}}-\frac{2 l(x)\left(l(x)-l_{b}\right)}{\mu+s+\gamma} A^{\frac{\mu+s+\gamma}{\gamma}}+\frac{\left(l(x)-l_{b}\right)^{2}}{\mu+s+2 \gamma} A^{\frac{\mu+s+2 \gamma}{\gamma}}\right\}
$$

with

* A solution $y$ to $h(x)=0$ is called simple if $\frac{d h}{d x}(y) \neq 0$. 


$$
A=\frac{l(x)-l_{J}}{\underline{l}(x)-l_{b}} .
$$

Hint: use $A$ as the integration variable in (3.3.7).

EXERCISE 3.3.4: Calculate $\psi\left(l_{b}\right)$ from condition (3.3.4b).

EXERCISE 3.3.5: Devise a strategy for finding a starting value such that a Newton iteration applied to (3.3.5) is guaranteed to converge. Hint: use the graphical interpretation of the Newton iteration and exploit the fact that $\pi_{x}(s)$ is a convex function of $s$.

EXERCISE 3.3.6: What can you tell about the dependence of $r$ on the other parameters of the model?

EXERCISE 3.3.7: What can you tell about the dependence of $x_{c}$ on the parameters of the model?

\subsection{Constant environments: reduction to an age-dependent problem}

The assumptions on the individual level imply that for constant food density there will be a fixed relation between size and age given by the solution to the equation for individual growth (3.1.3). Therefore we can also approach the problem of density independent Daphnia population growth from the angle of classical age dependent theory. By far the easiest way to do that is to start right from scratch, or more specifically from the considerations presented in subsection 3.1; and you are invited to do so yourself (possibly with some reference to IV.1, where we describe how to approach age dependent problems in general). In this subsection, however, we shall, for pedagogical reasons, show how the age dependent formalism can be derived from the more basic equation (3.2.1), as this allows us to introduce in a relatively painless manner the extremely useful mathematical concept of characteristic, which in this particular case is nothing but the trajectory of an individual in $\mathbb{R}^{+} \times \Omega$, where $\mathbb{R}^{+}$is our time set. Moreover, the actual calculations performed to effect the transformation are in many ways exemplary of the bookkeeping encountered in dealing with structured population models in general. The reward at the end of the road will be a straightforward biological interpretation of the crucial quantity $\pi_{x}(0)$. The greater gain, however, will be the acquired facility with some types of calculations which initially may seem forbidding, but really are quite easy provided you have had some practice.

The mathematical reasoning underlying the introduction of characteristics generally, starts by rewriting (3.2.1a) as

$$
\left(\frac{\partial}{\partial t}+g(x, l) \frac{\partial}{\partial l}\right) n(t, l)=-\left(g_{l}(x, l)+\mu\right) n(t, l),
$$

where

$$
g_{l}(x, l):=\frac{\partial}{\partial l} g(x, l)
$$

This then suggests considering $t$ and $l$ as functions of an independent variable $a$ such that precisely

$$
\frac{d}{d a}=\frac{d t}{d a} \frac{\partial}{\partial t}+\frac{d l}{d a} \frac{\partial}{\partial l}=\frac{\partial}{\partial t}+g(x, l) \frac{\partial}{\partial l}
$$

(the first equality being nothing but the chain rule for functions of more than one variable), as this allows us to write (3.4.1), and perforce (3.2.1a), as an ordinary differential equation

$$
\frac{d}{d a} n(t(a), l(a))=-\left(g_{l}(x, l(a))+\mu\right) n(t(a), l(a)),
$$

which can easily be solved explicitly (always assuming that we can find ways to provide an initial condition). The final step is to equate coefficients of like differential operators in (3.4.3) to arrive at

$$
\begin{aligned}
& \frac{d t}{d a}=1, \\
& \frac{d l}{d a}=g(x, l) .
\end{aligned}
$$

Solving (3.4.5) for various initial conditions ( $t, l)$ defines a family of curves in $\mathbb{R}^{+} \times \Omega$, such that through each point $(t, l) \in \mathbb{R}^{+} \times \Omega$ passes one and only one curve. These are the characteristics of (3.4.1) or equivalently (3.2.1a). 


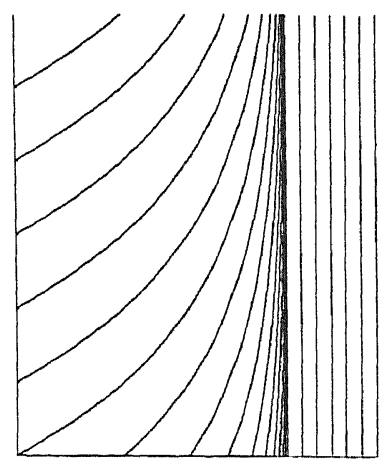

Figure 3.4.1. The characteristics of the Daphnia model

We shall now clarify the sense in which the characteristics can be interpreted as trajectories of individuals. (3.4.5a) tells us that $a$ and clock time proceed at the same speed, suggesting an interpretation as age. This suggestion is supported by the observation that (3.4.5b) is nothing but the differential equation for individual growth (3.1.3b). The only problem is choosing the appropriate initial conditions. At age zero we should have $l(0)=l_{b}$. So for the characteristics starting from the left hand boundary $\left\{\left(t_{0}, l_{b}\right) \mid t_{0}>0\right\}$ in figure 3.4.1 we can conventionally set $t(0)=t_{0}$ and $l(0)=l_{b}$, and interpret the characteristic starting from $\left(t_{0}, l_{b}\right)$ as the trajectory of an individual born at $t_{0}$. For the characteristics that start on the lower boundary $\left\{\left(0, l_{0}\right) \mid l_{0} \in \Omega\right\}$, we shall just set $t(0)=0$ and $l(0)=l_{0}$, and interpret $a$ as time since the start of the experiment, and the characteristic itself as the trajectory of an individual already present in the starting population, and having length $l_{0}$ at $t=0$. (Of course we could formally extend the characteristics into the region of negative time in order to keep the age interpretation (but only formally, individuals in the starting population may have experienced different feeding histories!), and we shall do so in III.4.2, but for our present purposes such a ploy would be counterproductive.) With these conventions the characteristics are given by

$$
t=t_{0}+a, \quad l=e^{-\gamma a}\left(l_{b}-\underline{l}(x)\right)+\underline{l}(x),
$$

for the characteristics which pass through the left hand boundary of $\mathbb{R}^{+} \times \Omega$, and

$$
t=a, \quad l= \begin{cases}e^{-\gamma a}\left(l_{0}-\underline{l}(x)\right)+\underline{l}(x) & \text { if } l_{0}<\underline{l}(x), \\ l_{0} & \text { if } l_{0} \geqslant \underline{l}(x),\end{cases}
$$

for the characteristics passing through the lower boundary.

The whole reason for introducing the concept of characteristic is that it allows us to get rid of the partial differential equation (3.4.1), or equivalently (3.2.1a), by integrating it along the characteristics, i.e. by solving the ordinary differential equation (3.4.4), the initial condition for (3.4.4) being provided by either the initial size distribution $n(0, l)$ for the characteristics defined by $(3.4 .6 \mathrm{~b})$ or the boundary condition $(3.2 .1 \mathrm{c})$, here rewritten as

$$
g\left(x, l_{b}\right) n\left(t, l_{b}\right)=b(t),
$$

where $b$ is the population birth rate for the characteristics defined by (3.4.6a). Substituting the explicit expression for $g_{l}(x, l)$ turns (3.4.4) into

$$
\frac{d n(t(a), l(a))}{d a}=\left\{\begin{array}{cc}
(\gamma-\mu) n(t(a), l(a)) & \text { for } l(a)<l(x), \\
-\mu n(t(a), l(a)) & \text { for } l(x) \leqslant l(a)
\end{array}\right.
$$

so that

$$
n(t, l)= \begin{cases}e^{(\gamma-\mu) a} n\left(t_{0}, l_{b}\right) & \text { for } l<L(t) \\ e^{(\gamma-\mu) a} n\left(0, l_{0}\right) & \text { for } L(t) \leqslant l<\underline{l}(x) \\ e^{-\mu a} n\left(0, l_{0}\right) & \text { for } \underline{l}(x) \leqslant l\end{cases}
$$

where

$$
L(a):=e^{-\gamma a}\left(l_{b}-\underline{l}(x)\right)+\underline{l}(x)
$$


is the individual growth curve. (3.4.9) relates the density, i.e. number of individuals per length unit, now at $l$ to either the density of newborns or the density of individuals in the starting population from which they have grown. The factor $e^{-\mu a}$ represents survival. The factor $e^{\gamma a}$ derives from the fact that we are dealing with a contracting conveyor belt. The more we approach $l(x)$ the more the conveyor belt contracts and so the more individuals have to be stowed away on the same amount of length.

To put (3.4.9) to work we still need to express $a$ and either $t_{0}$ or $l_{0}$ as functions of $t$ and $l$. To this end we introduce the function

$$
A(l):=L^{(-1)}(l)=\gamma^{-1} \ln \left(\frac{\underline{l}(x)-l_{b}}{\underline{l}(x)-l}\right),
$$

the age at which an individual first reaches length $l$. Then

$$
\begin{aligned}
& t_{0}=t-A(l), \quad a=t-t_{0} \text { for } l<L(t), \\
& a=t, \quad l_{0}=\left\{\begin{array}{lc}
L(A(l)-t) & \text { for } L(t) \leqslant l<l(x), \\
l & \text { for } l(x) \leqslant l .
\end{array}\right.
\end{aligned}
$$

EXERCISE 3.4.1: Express (3.4.12) verbally, in biological terms.

EXERCISE 3.4.2: Reexpress (3.4.12b) more explicitly.

By combining (3.4.9) and (3.4.12) with (3.4.7) we have found an explicit expression for $n(t, l)$, but in terms of the as yet unknown birth rate $b$. However, we also have available (3.2.1d) expressing the birth rate in terms of $n$. By combining these two expressions we end up with an equation for the unknown $b$ :

$$
b(t)=\frac{(1-\kappa) f(x)}{\omega w_{b}} \int_{L}^{\max (l, L(t))} l^{2} e^{(\gamma-\mu) a} \frac{b(t-a)}{\gamma\left(\underline{\left.l(x)-l_{b}\right)}\right.} d l+h(t)
$$

where $h$ represents all births from parents which were already present at $t=0$ :

$$
h(t)=\frac{(1-\kappa) f(x)}{\omega w_{b}} \int_{L(t)}^{l(x)} l^{2} e^{(\gamma-\mu) t} n\left(0, l_{0}\right) d l+\frac{1}{\omega w_{b}} \int_{\underline{l}(x)}^{\bar{l}(x)}\left(f(x) l^{2}-\zeta l^{3}\right) e^{-\mu t} n(0, l) d l .
$$

The integral in (3.4.13) can be rendered in a more intuitively appealing way by using $a=A(l)$ as the integration variable, using $d l / d a=-\gamma(l-\underline{l}(x))=\gamma e^{-\gamma a}\left(l(x)-l_{b}\right)$ :

$$
b(t)=\frac{(1-\kappa) f(x)}{\omega w_{b}} \int_{a}^{\max \left(a_{s}, t\right)} L^{2}(a) e^{-\mu(a)} b(t-a) d a+h(t)
$$

where

$$
a_{J}:=A\left(l_{J}\right) \text {, }
$$

the age at which an individual becomes reproductive. In words: the birth rate at $t$ equals the cumulation of the births from all individuals born at different times between time zero and now, which are still among the living, plus the births produced by the remains of the initial population.

Equation (3.4.15) is a so-called renewal equation (or linear Volterra integral equation of the convolution type). In V.2 we shall discuss a theorem telling that under fairly general conditions, one of which is that $h$ does not grow too ast, the solution to equations like (3.4.15) will grow exponentially for large $t$. The speed of exponential growth can be determined by substituting

$$
b(t)=c e^{r t}(1+o(t))
$$

in (3.4.15), dividing both sides by $c e^{r t}$ and formally taking the limit for $t \rightarrow \infty$ (IV.2 will give a slightly more rigorous but considerably less appealing rendering), to arrive at the characteristic equation

$$
1=\frac{(1-\kappa) f(x)}{\omega w_{b}} \int_{a}^{\infty} L^{2}(a) e^{-\mu a} e^{-r a} d a .
$$

Which brings us full circle.

EXERCISE 3:4.3: Combine (3.4.17) with (3.4.9), (3.4.12a) and (3.4.7) to rederive the stationary length distribution $\psi$. 
From its derivation it should be intuitively clear that (3.4.18) can be nothing but the characteristic equation (3.3.5) in a different disguise. Therefore its right hand side provides another way of calculating $\pi_{x}$. This can also be checked algebraically. However, the form in which $\pi_{x}$ is written in (3.4.18) gives a much better clue to the biological interpretation of the condition $\pi_{x}(0) \gtreqless l$. Since $\mu$ is the (constant) death rate, $e^{-\mu a}$ is precisely the probability that an individual survives to age $a$. The factor $\left(\omega w_{b}\right)^{-1}(1-\kappa) f(x) L^{2}(a)$ is the fertility of an individual aged $a$. So $\pi_{x}(0)$ equals the number of offspring a newborn individual is expected to bear. When this number is greater than one the population grows, otherwise it goes extinct.

A small sleight of hand and concentrating on the borderline case $\pi_{x}(0)=1$ also makes it possible to interpret the characteristic equation: $\pi_{x}(s)$ equals the expected number of offspring per individual if we harvest a population by randomly removing individuals at a rate $s$, i.e. replacing $\mu$ by $\mu+s$. If the harvesting rate precisely equals the natural population growth rate the population size should remain constant, which in turn is equivalent to the expected number of offspring per individual in the harvested population being precisely equal to one.

EXERCISE 3.4.4: Check algebraically that indeed (3.4.18) is just (3.3.5) written in a different form.

EXERCISE 3.4.5: It is also possible to attack age dependent problems by interpreting age as an $i$-state variable. Write down the $p$-equations, direct from a priori considerations. ${ }^{*}$ Also rederive $(3.2 .1)$ from the age dependent equations and the function $L$ which expresses $l$ in terms of $a$.

\subsection{Variable environments}

The simple hypothesis of constant food density will seldomly be fulfilled in practice except for very short periods. Food densities fluctuate due to external causes and as a result of the feeding activity of the Daphnia. In simple laboratory systems the external causes are kept constant, so only the feeding activity of the population has to be taken into account. The simplest assumption we can make about the food dynamics is

$$
\frac{d x}{d t}=k(x)-f(x) \int_{\Omega} l^{2} n(t, l) d l,
$$

where $k$ describes the dynamics of the food population in the absence of Daphnia, and the second term is precisely the amount of food ingested by all individuals together. For $k$ we may e.g. choose

$$
\begin{aligned}
& k(x)=\alpha-\beta x, \\
& k(x)=\alpha x-\beta x^{2} .
\end{aligned}
$$

The first assumption corresponds to a constant inflow of fresh, nonreprodıcing, food particles combined with a constant food deterioration, e.g. through sinking (or dilution in a flow culture). The second assumption corresponds to logistic growth of an unstructured food population. More generally $k$ may be assumed to be positive for $0<x<x_{e}$ and negative for $x>x_{e}$ for some parameter $x_{e}$.

If we consider the combined food-Daphnia system the first step will be the determination of any possible equilibria $\hat{n}, \hat{x}$. Clearly $\hat{n}(l)=0, \hat{x}=x_{e}$ is an equilibrium: no Daphnia present and the food density at its unexploited equilibrium. This is usually called the trivial equilibrium or also the boundary equilibrium. The results of the previous sections imply that in any non-trivial (or internal) equilibrium the food density $\hat{x}$ must be such that

$$
\pi_{\hat{x}}(0)=1 \text {, }
$$

since, given a constant food density $\hat{x}$, the Daphnia numbers should remain constant. In subsection 3.3 we already found that this equation has a unique solution

$$
\hat{x}=x_{c}
$$

provided $\pi_{\infty}(0)>1$. We conclude that at the nontrivial equilibrium the food density is solely determined by the Daphnia dynamics, independent of the details of the food dynamics as expressed in the function $k$. The corresponding equilibrium Daphnia density is necessarily of the form

$$
\hat{n}(l)=c \psi_{x_{c}}(l),
$$

where $\psi_{x_{c}}$ is the (normalized) stable size distribution (at the critical density $x_{c}$ given by (3.3.4)), and $c$ is a constant which has to be chosen such that the right hand side of (3.5.1) is zero for $x=\hat{x}=x_{c}$ and $n(t, l)=\hat{n}(l)$.

$$
c=\frac{k\left(x_{c}\right)}{f\left(x_{c}\right) \int_{l_{b}}^{l\left(x_{c}\right)} l^{2} \psi_{x_{c}}(l) d l} .
$$


But of course we also require that $c>0$, and therefore a necessary and sufficient condition for the existence of a nontrivial equilibrium is that

$$
x_{c}<x_{e} \text {. }
$$

Condition (3.5.8) also emerges from a consideration of the stability of the boundary equilibrium. If we add a very small number of Daphnia to a food population which is at its own dynamical equilibrium $x_{e}$, the dynamics of the Daphnia will, initially at least, conform approximately to the assumption of a constant food density. The results from section 3.3 then tell us that the Daphnia population will go extinct whenever $x_{e}<x_{c}$ and will start to grow when $x_{c}<x_{e}$. In IV.4.2 this argument is extended to include the borderline case $x_{e}=x_{c}$ : there it is proved that the boundary equilibrium is globally attractive if $x_{0} \leqslant x_{c}$. Our results till now therefore can be summarized by saying that there exists a unique internal equilibrium whenever the autonomous food equilibrium is invadable, and when this is not the case no Daphnia population can maintain itself.

The stability of the internal equilibrium is a more complicated matter. The general results from bifurcation theory, discussed in chapter VI, suggest that the internal equilibrium will be asymptotically stable for parameter values which are such that $x_{e}$ is but slightly larger than $x_{c}$.
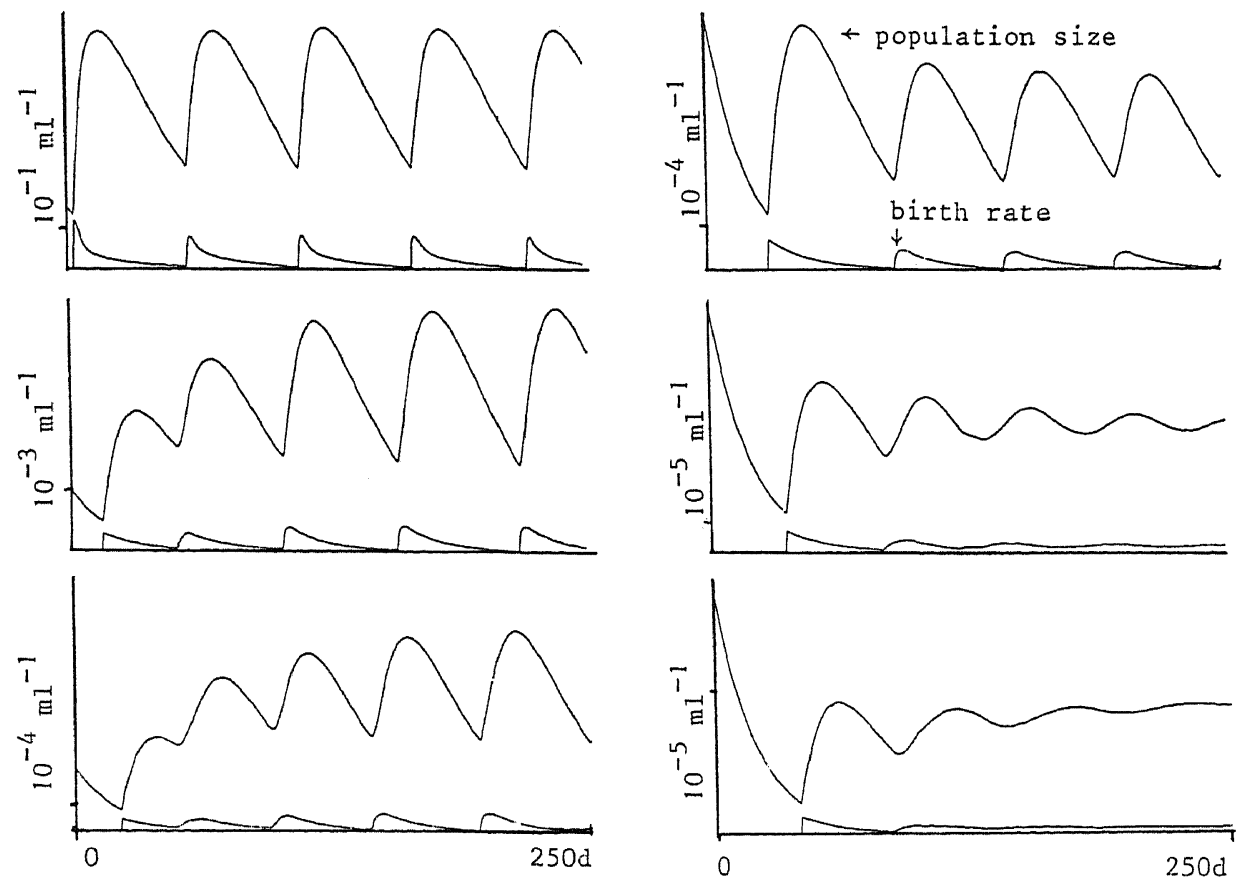

Fig. 3.5.1: Food regulated Daphnia dynamics: numerical results for a flow culture with continuous feeding (food dynamics (3.5.2)) for various values of the feeding rate. Upper trace: total population size. Lower trace: birth rate Parameters $\quad l_{b}=.8 \mathrm{~mm}, \quad l_{J}=2.5 \mathrm{~mm}, \quad l_{m}=\kappa \nu / \zeta=6.6 \mathrm{~mm}, \quad \gamma=\zeta / 3 \eta=.17 d^{-1}$, $\lambda_{m}=(1-\kappa) l_{m} \nu / \omega w_{b}=10 d^{-1}, \quad \kappa=.3333, \quad \xi=.7 \times 10^{-6} \mathrm{ml} / \mathrm{cell}, \quad \nu=1.8 \times 10^{6} \mathrm{cell} / \mathrm{mm}^{2}$, $\beta=.5 d^{-1}, \alpha=10^{6}, 5 \times 10^{4}, 4.5 \times 10^{4}, 4.37 \times 10^{4}, 4.36 \times 10^{4} \mathrm{cell} / \mathrm{ml} . \mathrm{d}$ (from top to bottom, left to right ).

The numerical results shown in figure 3.5.1 indicate that for other parameter values it may become unstable and that asymptotically stable oscillations result. The suggestion is that the transition comes about through a so-called Hopf bifurcation, but before such a result can be proven rigorously, the theory of structured population models still needs to be developed a great deal further.

EXERCISE 3.5.1: Show that two species of Daphnia cannot coexist in stable equilibrium unless they have equal values of $x_{c}$. If this is not the case which species would you tip as winner?

* EXERCISE 3.5.2: Even if there were no numerical results one would suspect that any transition to instability would be 
through a Hopf bifurcation. Why? Hint: Refer to the results from bifurcation theory sketched in VI.1.

\subsection{Summary}

In this section we approached the problem of modelling the population dynamics of ectothermic animals on the basis of simple physiological considerations, concentrating on "size" as the major $i$-state variable.

We started with some simple energetic and dimensional considerations on the individual level along the lines laid out by KOOIJMAN \& METZ (1984). This way we arrived at a simple model for individual behaviour, taking account of feeding, growth, basal metabolism, reproduction (after reaching adult size), and starvation, all in dependence on individual length (or equivalently weight as we assumed selfsimilar growth), and food density. From this $i$-model we then derived a set of $p$-equations which formed the basis of our further considerations.

At a constant food density the population after some time will grow exponentially, with rate constant $r$, while the size distribution stabilizes. An explicit expression for the stable size distribution can be given once $r$ is known, but $r$ itself has to be calculated numerically from the characteristic equation. $r$ increases with the food density $x$, being positive only for $x$ above some critical value $x_{c}$ which again can easily be calculated numerically.

At a constant food density, size is uniquely related to age, implying that then there should exist an equivalent age based model, and we have gone to some effort to derive this model from the equations of the size based model, mainly in order to demonstrate some useful mathematical concepts and techniques, in particular integration along characteristics.

In the final subsection we considered a dynamic food source, the consumption by the animals being an essential component of its dynamics. When the autonomous food equilibrium $x_{e}$ is larger than $x_{c}$ there exists a unique nontrivial equilibrium. Otherwise the consumers go extinct. At equilibrium the food density necessarily equals $x_{c}$ and the size distribution of the consumers is given by the stable size distribution of the linear problem studied previously, for $r=0$. Although the non-trivial equilibrium may be stable we found numerically that often it is replaced by a limit cycle as the stable attractor.

\section{The cell size distribution}

In the final example of this chapter we shall turn to unicellular organisms. From the very beginning of their science microbiologists have been interested in population growth curves i.e. the number of individuals in a culture plotted as a function of time. Microorganisms also provided the conventional testing ground for the first theoretical developments in population modelling. These early models were all phrased in terms of ordinary differential equations, as are most models today, without taking into account any aspect of the population structure, even if it was recognized that population growth characteristics, at least initially, tend to depend on the origin (and therefore the composition) of the inoculum.

Clearly individual cells do not divide purely at random as is assumed in the conventional differential equation models. Such traits as age, size, weight and biochemical composition all contribute to determining when the next cell division will occur. A natural approach, and the one advocated here, is to seek to describe the cell state as completely as is feasible by a limited number of numerical variables, and to derive equations which enable us to deduce relations between, on the one hand, the statistical and dynamical behaviour of populations of cells, and, on the other hand, the physiological processes which take place in the individual cells. Moreover, nowadays sophisticated equipment enables us automatically to gather statistical data on various aspects of population composition, thus putting those models on a level beyond that of mere figments of imagination.

At the end of the sixties at least three groups of theoreticians have, independently, derived such equations (BELL \& ANDERson, 1967; Fredrickson, Ramkrishna \& TsuchiYa, 1967; Sinko \& Streifer, 1967). They philosophised about the structure of mathematical models, did computer simulations and fitted experimental data. Yet after this strong start the development stagnated again, probably due to a combination of causes. Routine automated data gathering as well as routine computing were much less well developed than they are today. But the main factor probably was that the equations were so far removed from the available mathematical tools of the time, that there was no incentive for any concerted effort from the mathematical and theoretical biological research communities. All this has changed considerably by now, and the theory of structured cell population models is showing distinct signs of a renewed bloom.

Below we shall present a relatively simple model, in which cell size is the only $i$-state variable. This model will also be the main example around which the next chapter, dealing with the mathematical toolkit, revolves. In chapter $\mathrm{V}$ a 
more complicated model taking account of age as well will be developed, and chapter VI also contains two applications to cell population dynamics. In part B Voorn \& Koch consider various statistical aspects of size structured cell populations and Kooijman explores the consequences of extending the $i$-state description to account for reserves.

\subsection{The dynamics of individuals}

Clearly the step from no population structure to a realistic structure, in which, say, all available knowledge about the biochemical processes within the cell is taken into account, is much too large. We therefore take an intermediate position and assume that the state of a cell is completely characterized by one quantity $x$ which moreover obeys a physical conservation law, in the sense that at cell division it is divided up among the daughters. One can think of $x$ as meaning: the length of oblong cells, the volume of a cell, dry mass, nitrogen content etc. But obviously not age. We shall call $x$ size.

Given our choice of the $i$-state a cell has only three behavioural possibilities: growing, dividing and dying (including wash-out in a chemostat). Any other processes like the merging of cells as part of some sexual form of reproduction we simply leave out of the account.

As in Daphnia we shall assume growth to be completely deterministic, occurring at a rate $V$ :

$$
\frac{d x}{d t}=V(x), \quad V>0 \text {. }
$$

Of course $V$ will also depend on environmental variables such as temperature and nutrient concentration, but we shall only express this in our notation when the variation of these variables actually will be accounted for in the model. Otherwise the environment will simply be assumed constant.

Fission we shall treat as a stochastic process. In fact our description of the state by only one variable is by necessity rather poor. The best we may hope for is that it is the main variable determining the occurrence of division. The observed variability in the size at division we account for by building a stochastic element into the model. Interestingly enough it turns out that this non-mechanistic artifice needs further specification when the growth rate is subject to environmental variation (DIEKMANN, LAUWERIER, ALDENBerg \& METZ, 1983). Several possibilities present themselves. We shall only consider what in a sense are the two extreme cases:

(i) We postulate that the rate, or probability per unit of time, at which cells of size $x$ undergo fission is given by a function

$$
b(x) \geqslant 0
$$

which we assume to be known.

(ii) We postulate that each cell has a stochastically predetermined size at which fission has to occur, independent of any characteristics of its forebears, provided the cell does not die before that time. This may also be expressed more graphically by saying that division occurs when cell size reaches a stochastic threshold (cf fig. 4.1.1). The probability density of this hypothetical "size at division precluding death" will be denoted as $\phi_{b}$, i.e. if we consider a large population of cells all starting life at so small a size that they cannot possibly divide yet, a fraction

$$
\int_{x_{1}}^{x_{2}} \phi_{b}(x) d x
$$

will divide between sizes $x_{1}$ and $x_{2}$, provided we have eliminated all possible causes of death. (In the terminology of PAINTER \& MARR (1968), $\phi_{b}$ is called the probability density for the size at division in a sample of newborn cells; the supposition behind this term clearly being that a) even the largest newborn cells are so small that any smaller cell cannot divide, and b) no cells ever die (see exercise 4.1.5).)

In order to investigate the relation between these two possible paradigms we imagine a cohort of $N_{0}$ immortal cells all having size $a$ at time $t=0$, where $a$ is the minimal size at which division can possibly occur. As time increases their size changes according to

$$
\frac{d x}{d t}=V(x), \quad x(0)=a,
$$

and their number according to

$$
\frac{d N}{d t}=-b(x(t)) N, \quad N(0)=N_{0} .
$$




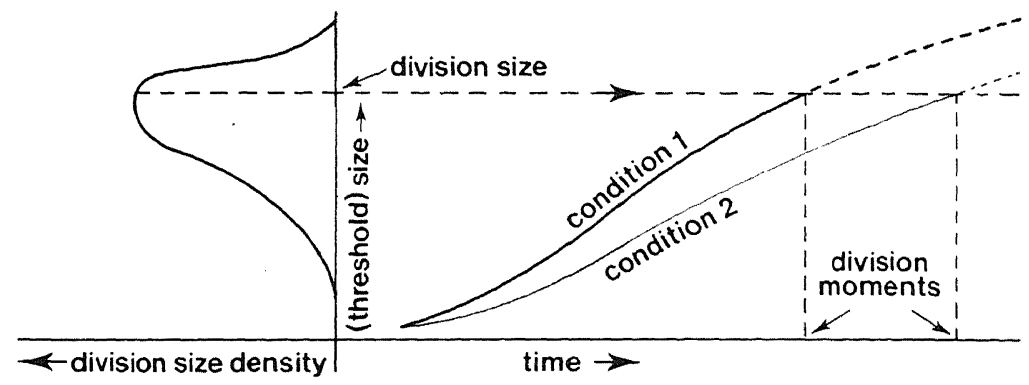

Fig. 4.1.1 The division on crossing a stochastic size threshold paradigm. Under condition 2 the ages at cell division are larger than under condition 1 but the distribution of the size at division remains the same.

Hence

$$
N(t)=N_{0} \exp \left\{-\int_{0}^{t} b(x(\tau)) d \tau\right\} .
$$

Using $\xi=x(\tau)$ as a new integration variable we see that

$$
N_{0} \exp \left\{-\int_{a}^{x} \frac{b(\xi)}{V(\xi)} d \xi\right\}
$$

cells reach size $x$. But this number should equal

$$
N_{0}\left(1-\int_{a}^{x} \phi_{b}(\xi) d \xi\right)
$$

And by differentiating these two expressions we deduce that

$$
\phi_{b}(x)=\frac{b(x)}{V(x)} \exp \left\{-\int_{a}^{x} \frac{b(\xi)}{V(\xi)} d \xi\right\},
$$

and, conversely, by differentiating their logarithms,

$$
b(x)=V(x) \frac{\phi_{b}(x)}{1-\int_{a}^{x} \phi_{b}(\xi) d \xi} .
$$

So, if $V$ does not depend either explicitly or implicitly on time, (i) and (ii) are just two different ways of doing the bookkeeping. But if $V$ does vary with time, then at least one of the two functions $b$ or $\phi_{b}$ has to be nonconstant as well. Fig. 4.1.2 depicts the essential dynamical difference between the two mechanisms. In that case we have to make a choice: what is the intrinsic characteristic of the cell, $b, \phi_{b}$ or still something else? This need not bother us for the time being as in most of this section and chapter II we shall assume that $V$ is just a fixed function of $x$ only. However, near the end of both the distinction will become a crucial modelling ingredient.
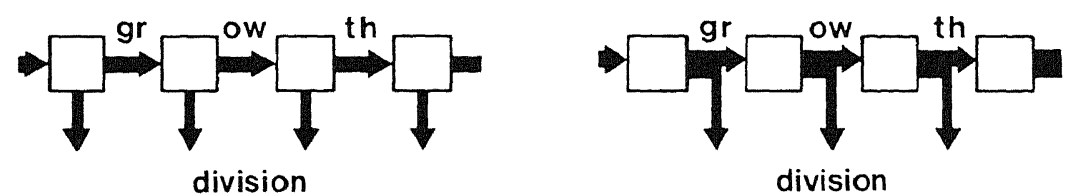

Fig. 4.1.2 The essential dynamical difference between division mechanisms (i) and (ii). 
We complete the description of the division process by assuming that division always is into two exactly equal daughters, and our description of individual behaviour by assuming that deaths occur randomly at a possibly size dependent rate

$\mu(x) \geqslant 0$.

EXERCISE 4.1.1: What is the probability that a cell having birth size $a$, in which division is inhibited but that is otherwise the same, survives till age $\tau$ ? That it dies before $\tau$ ? What is the probability density of its age at dying?

EXERCISE 4.1.2: What is the probability that the cell of the previous exercise survives till size $x$ ? The probability density of its size at dying?

EXERCISE 4.1.3: What is the probability that a cell that may both divide and die ever reaches age $\tau$, size $x$ ? Assume again that it has birth size $a$.

EXERCISE 4.1.4: What is the probability that the cell from the previous exercise divided between ages $\tau$ and $\tau+d \tau$ ? What is the probability that it dies before reaching division?

EXERCISE 4.1.5: What is for the cells of the previous exercise the probability density of the size at division (counting only cells that reach division)?

EXERCISE 4.1.6: Assume that there is a lower limit $a$ to the size at division, and that $a$ is larger than the size of any newborn cell. What is the expected number of daughters reaching size $a$, of a cell that itself at this moment passes size a?

\subsection{Formulation of the $p$ equations}

Just as for Daphnia the size density will fulfill the role of $p$-state. We shall denote it as $n$, i.e. $\int_{y}^{z} n(t, x) d x$ is the number of cells with sizes between $y$ and $z$ at time $t$. Drawing up the balance of growth, death and division during an arbitrarily small time interval, like we did in the previous sections, we find

$$
\frac{\partial}{\partial t} n(t, x)=-\frac{\partial}{\partial x}(V(x) n(t, x))-\mu(x) n(t, x)-b(x) n(t, x)+4 b(2 x) n(t, 2 x) .
$$

The factor 4 in the birth term may come as a bit of a surprise. The first of the two 2's from which it is made up comes from the cells dividing into two. The second 2 derives from the fact that cells born into the interval $(x, x+d x)$ come from parents in the twice as long interval $(2 x, 2(x+d x))$. So when the mass is lifted from the conveyor belt, doubled and deposited again it also is compressed sideways by a factor one half resulting once again in a doubling of its height.

The next step, as usual, is to see whether any side conditions have to be imposed to arrive at a well-defined mathematical problem. This also means that we have to specify more carefully what the individual state space $\Omega$ looks like. Let us assume that there exists a smallest size $a$ at which fission can occur, i.e.

$$
a=\inf \{x \mid b(x)>0\}
$$

is positive. Then $\frac{1}{2} a$ is the smallest size a cell can possibly have. Integration of (4.2.1a) with respect to $x$ yields

$$
\frac{d}{d t} \int_{\frac{1}{2} a}^{\infty} n(t, x) d x=V\left(\frac{1}{2} a\right) n\left(t, \frac{1}{2} a\right)-\int_{\frac{1}{2} a}^{\infty} \mu(x) n(t, x) d x+\int_{a}^{\infty} b(x) n(t, x) d x .
$$

In words: the number of cells changes due to resp. (i) cells growing beyond $\frac{1}{2} a$, (ii) cells that die, and (iii) cells that divide (at each division cell number increases by one). We want the first term to be zero (indeed, where would those cells come from?) Since $V\left(\frac{1}{2} a\right)>0$ we have to impose the boundary condition

$$
n\left(t, \frac{1}{2} a\right)=0 \text {. }
$$

We shall also assume that there exists a maximal size $x_{\max }$, in the sense that cells will divide with probability one before reaching $x_{\max }$, in accordance with accepted biological wisdom. Without loss of generality we may set $x_{\max }=1$. 
(We can always adjust the unit of size to suit our purpose.) How should this biological assumption be expressed in our mathematical formulation? In terms of the probability density $\phi_{b}$ our assumption is equivalent to

$$
\int_{a}^{1} \phi_{b}(x) d x=1
$$

which, in view of the equality of $(4.1 .4 \mathrm{a}, \mathrm{b})$, in turn is equivalent to $b$ having a non-integrable singularity at $x=1$.

Our two assumptions of a minimal size $a$ at division and a maximal size $x_{\max }=1$, imply that $\Omega=\left(\frac{1}{2} a, 1\right)$. As $n$ only "lives" on $\Omega$ the term $4 b(2 x) n(t, 2 x)$ in (4.2.1a) is undefined for $x>\frac{1}{2}$ but for convention 2.5.3, which we repeat here as

CONVENTION 4.2.1: If a transformed argument falls outside $\Omega$ we shall assume that the term in which it occurs equals zero.

REMARK 4.2.2: Also (4.2.1b) should be interpreted as $\lim _{x \downarrow \frac{1}{2} a} n(t, x)=0$.

Our assumptions on the $i$-level also, somewhat unexpectedly, preclude us from prescribing just any size distribution on $\left(\frac{1}{2} a, 1\right)$ as initial condition for (4.2.1)! Cells with larger sizes become increasingly scarce relatively, and therefore any size density compatible with our basic mechanism will have to taper off near $x=1$. In the next chapter it will be shown that the collection of size densities $\nu(x)$ such that $\nu(x) /\left(1-\int_{\frac{1}{2} a}^{x} \phi_{b}(\xi) d \xi\right)$ is bounded for $x \uparrow 1$ is invariant under (4.2.1). Confining the attention to compatible size densities also prevents the birth term $4 b(2 x) n(t, 2 x)$ growing out of bounds for $x \uparrow \frac{1}{2}$ (provided $\phi_{b}$ is bounded). It precisely is this sort of subtlety which makes the theory of structured populations so complicated mathematically, but also so enticing.

EXERCISE 4.2.3: Can you also derive (4.2.1b) from a heuristic conveyor belt type argument?

EXERCISE 4.2.4: Multiply (4.2.1) with $x$ and integrate over $x$ to derive a differential equation for the "total biomass", and interpret the result in biological terms. What happens to the last two terms, and why?

EXERCISE 4.2.5: Assume in the previous exercise that $\mu$ is constant and that $V(x)=\alpha x$. How does the total biomass develop over time? (Unfortunately the special case $V(x)=c x$ has rather unpleasant properties in other respects; see below.)

EXERCISE 4.2.6. Another way to guarantee that the cells divide before reaching size $x_{\max }=1$, is to assume that $V(x) \downarrow 0$ for $x \uparrow 1$. Does this assumption have any consequences for $b$ ? How can one distinguish experimentally between the two assumptions using the (population) distribution of the time until division. (This quantity is relatively easy to obtain experimentally, such in stark contrast to the individual growth curve)?

\subsection{The stable cell size distribution}

Equation (4.2.1) is dealt with at great length in chapter II. Therefore we shall confine ourselves here to giving a short heuristic introduction to the main results, stressing the biological interpretation. In this subsection we shall concentrate on the large time behaviour for constant environments (i.e. we assume $V$ to depend on $x$ only), in subsection 4.4 we shall discuss how the results from the present subsection can be used to make inferences about individual behaviour from observations on the population level, and in subsection 4.5 we shall consider a specific case of population regulation through resource limitation.

Proceeding by analogy with the previous two examples we substitute a trial solution $n(t, x)=e^{k t} \lambda(x)$ in $(4.2 .1)$ (again we use the notation of PAINTER \& MARR (1968)), and find

$$
\frac{d V(x) \lambda(x)}{d x}=-(k+\mu(x)) \lambda(x)-b(x) \lambda(x)+4 b(2 x) \lambda(2 x) .
$$

For $x>\frac{1}{2}$ the last term drops out, and (4.3.1) becomes an ordinary differential equation which we can solve provided we prescribe some arbitrary initial condition, at $x=\frac{1}{2}$. Given $\lambda$ on $\left[\frac{1}{2}, 1\right)$ we can then work our way backwards and solve $\lambda$ on $\left(\max \left\{\frac{1}{2} a, \frac{1}{4}\right\}, \frac{1}{2}\right)$ and so on till we have filled $\Omega$. For the ease of presentation we shall from now on 
assume

$$
a \geqslant \frac{1}{2}
$$

so that we only have to make one retrograde step. (The general case is treated in great detail in HEIJMANS (1985b)) From the requirement that $\lambda$ satisfies the boundary condition

$$
\lambda\left(\frac{1}{2} a\right)=0
$$

it then is found that $k$ should satisfy the characteristic equation

$$
\pi(k)=1
$$

with

$$
\pi(s)=2 \int_{a}^{1} \frac{b(\xi)}{V(\xi)} \exp \left[-\int_{\xi / 2}^{\xi} \frac{s+\mu(\eta)+b(\eta)}{V(\eta)} d \eta\right] d \xi
$$

and (up to multiplication with an arbitrary constant)

$$
\lambda(x)=\frac{\rho(x)}{V(x)} \exp \left[-\int_{a / 2}^{x} \frac{k+\mu(\xi)+b(\xi)}{V(\xi)} d \xi\right]
$$

with

$$
\rho(x)=\left\{\begin{array}{lc}
1 & \text { for } \frac{1}{2} \leqslant x \leqslant 1 \\
\left.2 \int_{a}^{2 x} \frac{b(\xi)}{V(\xi}\right) \exp \left(-\int_{\xi / 2}^{\xi} \frac{k+\mu(\eta)+b(\eta)}{V(\eta)} d \eta\right) d \xi & \text { for } \frac{1}{2} a \leqslant x \leqslant \frac{1}{2} .
\end{array}\right.
$$

(Note that the relation $\pi(k)=1$ ensures that $\rho$ is continuous at $x=\frac{1}{2}$.)

REMARK 4.3.1: Instead of integrating backwards from $x=\frac{1}{2}$ we could also have integrated forwards from $x=\frac{1}{2} a$ using (4.3.1b) as initial condition. (4.3.3) then results from the requirement that $\lambda$ be continuous at $x=\frac{1}{2}$.

Since the functions $V, \mu$ and $b$ are nonnegative the function $\pi$ is strictly decreasing on the real axis. Moreover $\pi(-\infty)=+\infty$ and $\pi(+\infty)=0$. We conclude that there indeed exists precisely one simple real solution which we have chosen to call $k$. Given expressions for $V, b$ and $\mu$ it is relatively easy to determine $k$ numerically (e.g. by Newton iteration). As in the Daphnia case the sign of $k$ can easily be determined from the relation

$$
\pi(0) \geqslant 1 \Leftrightarrow k \geqslant 0 \text {. }
$$

INTERLUDE 4.3.2: $A$ biological interpretation of the quantities $\pi(0)$ and $\pi(s)$.

Since $a \geqslant \frac{1}{2}$ and size at birth $x_{b} \leqslant \frac{1}{2}$ each cell needs to pass size $x=a$ before it can possibly divide. Hence the average contribution of an arbitrary cell passing size $x=a$ to the next generation of cells can be measured by counting how many of its daughters on the average reach size $x=a$ themselves. This quantity is made up of the following components:

(i) he probability that a potential mother reaches size $\xi$ is $\exp \left(-\int_{a}^{\xi} \frac{\mu(\eta)+b(\eta)}{V(\eta)} d \eta\right)$

(ii) The probability per unit of size that it divides at size $\xi$, given that it has reached that size is $b(\xi) / V(\xi)$ (the factor $1 / V(\xi)$ converts probability per unit of time to probability per unit of size). The number of daughters is exactly 2 and each has size $1 / 2 \xi$.

(iii) The probability that a cell born with size $1 / 2 \xi$ safely grows up to size $a$ is $\exp \left(-\int_{\xi / 2}^{a} \frac{\mu(\eta)}{V(\eta)} d \eta\right)$.

By integrating over all possible values of the size at division $\xi$ we arrive at the interpretation: $\pi(0)$ is the average number of offspring of cells sampled at size $a$ growing up to at least size $a$ again.

To interpret $\pi(s)$ for general $s$ we observe that in (4.3.3) $s$ occupies exactly the same position as $\mu$, allowing us to interpret it as an additional death rate which can be imposed on the population in order to induce exact replenishment: $\pi(s)=1$. $($ Compare the argument at the end of section 3.4.) 
EXERCISE 4.3.3: Calculate $\pi(0)$ for the special case $\mu(x)=0$ and interpret the answer. Hint: Use that $b(x)=0$ for $x<a$.

Now that we have found a stable size distribution we should ask the convergence question: will the population state, after suitable normalization, converge to the stable size distribution, and will the population size settle on asymptotic exponential growth, independent of the initial condition (the composition of the inoculum)? Thus far we have ducked this question and for very good reasons. The answer turns out to be : it depends ; we know an (important) special case where it doesn't! More precisely:

(i) If $V(2 x) \neq 2 V(x)$ for some $x \in\left(\frac{1}{2} a, \frac{1}{2}\right)$ then for $t \rightarrow \infty$

$$
e^{-k t} n(t, x) \rightarrow C \lambda(x)
$$

where $C$ depends on the initial condition (in chapter II we show how $C$ can be computed; an explicit expression for $C$ may be found in Diekmann (1985)).

(ii) If, however, $V(2 x)=2 V(x)$ for all $x \in\left(\frac{1}{2} a, \frac{1}{2}\right)$ then

$$
e^{-k t} n(t, x) \rightarrow p\left(t-\int_{a / 2}^{x} \frac{d \xi}{V(\xi)}\right) \lambda(x)
$$

where $p$ is a periodic function, which depends on the initial condition, with period

$$
\int_{a / 2}^{a} \frac{d \xi}{V(\xi)}
$$

(in chapter II we show how $p$ can be computed).

And the exceptional case (ii) is not just a bizarre counterexample living only in the feverish imagination of mathematicians: the expression for $V$ most often encountered in the microbiological literature, $V(x)=\alpha x$, precisely belongs to this class! (Exercise 4.2.5 shows though that for this special case total biomass behaves quite nicely, even if cell number doesn't.)

In hindsight there is a simple biological argument, due to BELL \& ANDERsON (1967), who were the first to notice the non-convergence problem, which makes the special case $V(2 x)=2 V(x)$ easy to understand. Consider two cells $A$ and $B$ which have equal sizes at $t=0$, and assume that $A$ splits immediately into two daughters $a_{1}$ and $a_{2}$ and $B$ only splits at $t=t_{1}>0$ into $b_{1}$ and $b_{2}$. During the time interval $\left[0, t_{1}\right), B$ grows twice as fast as each of the $a$ 's. So at $t_{1}$ the $a$ 's and $b$ 's have exactly equal size! The relation "equal size" therefore is hereditary, extending over the generations, and therefore many properties of the initial condition will stay manifest over time.

The Bell \& Anderson explanation also immediately points to a "biological resolution" of the non-convergence problem. The anomaly apparently hinges on a combination of the two contingencies that (i) $V(2 x)=2 V(x)$ for all $x$ in $\left(\frac{1}{2} a, \frac{1}{2}\right]$, and (ii) cells split into two exactly equal parts. Therefore one expects that as soon as either (i) or (ii) is replaced by a different assumption the trouble will disappear. As the case $V(x)=\alpha x$ clearly is an important special case the next thing to do is to see whether modifying (ii) is of help. And indeed, Heismans (1984b) has proved that when a mother of size $x$ can split into two daughters of sizes $q x$ and $(1-q) x$, where $q$ is a random variable with density $d(q)$ independent of $x$, convergence to a stable size distribution occurs even when $V(2 x)=2 V(x)$. (See also exercise 4.3.6 and exercise II.11.3) So clearly our quandary depended on the unlucky combination of two simplifying assumptions. Yet both assumptions seem to be met to a good approximation in many practical cell populations. In such cases the present model predicts slow convergence towards a stable distribution (in the limiting case there is no convergence at all). A rough estimate of the relaxation time gives for a standard deviation of $q$ equal to 0.01 a relaxation time of 60 generations versus $2 \frac{1}{2}$ generations for $\mathrm{s} \mathrm{d} q=0.05$.

REMARK 4.3.4: If the probability density $d$ is highly peaked near $q=\frac{1}{2}$, formulae (4.3.3) and (4.3.4) stil will give a good approximation to the population growth rate $k$ and the stable size distribution $\lambda$, even for the case $V(x)=\alpha x$. But we have to interpret $\lambda$ given by (4.3.4) as the limit of the stable size of the more complicated model for the variance in $q$ going to zero, and not as the limiting size distribution of the simplified model for $t \rightarrow \infty$.

The common observation in cell cultures, where environmental conditions are kept constant by regularly changing the culture medium, is that after a certain initial time the normalized size distribution hardly changes, while numbers and other relevant quantities (total biomass, amount of DNA) grow exponentially. Cell biologists then say that the culture is in steady state or in a state of balanced exponential growth (see PAINTER \& MARR (1968) for a precise description of the terminology). So here clearly is a case indeed where our results (4.3.3) and (4.3.4) are highly relevant. 
* EXERCISE 4.3.5: Equation (2.5.5a) (the equation for the $p$-state of the invertebrate predator), with boundary condition $\left(2.5 .5 \mathrm{~b}^{\circ}\right)$, also allows an exceptional class of digestion rates $f$ for which there is no convergence to a stable stationary solution. (Clearly $f(s)=-$ as does not belong to this category). Could you guess which ones? Hint: In the predator model jumps were additive in the $i$-state variable, in the cell growth model multiplicative, and the anomaly apparently has to do with a relation between the nature of the continuous drift ( $f$ or $V$ ) and that of the jumps.

EXERCISE 4.3.6: Show that the unequal division model referred to above leads to the equation

$$
\frac{\partial}{\partial t} n(t, x)=-\frac{\partial}{\partial x}(V(x) n(t, x))-\mu(x) n(t, x)-b(x) n(t, x)+2 \int_{x}^{1} \frac{d(q)}{q} b\left(\frac{x}{q}\right) n\left(t, \frac{x}{q}\right) d q .
$$

Check that necessarily $d(q)=d(1-q)$, i.e. $d$ is symmetric about $q=\frac{1}{2}$. Moreover, check that the equation (4.2.1a) is recovered by formally putting $d(q)=\delta\left(q-\frac{1}{2}\right)$ where $\delta$ is the Dirac delta function(al). Exercise II.11.3 contains further information about (4.3.9).

EXERCISE 4.3.7: Show by integration of equation (4.3.1a) that $k \geqslant 0$ when $b(x) \geqslant \mu(x)$ for $x \in \Omega$ (which presupposes that $\mu(x)=0$ for $x \in\left(\frac{1}{2} a, a\right]$, i.e. preparing for division may be risky, but deaths of pre-division cells can be neglected) and $k \leqslant 0$ when $b(x) \leqslant \mu(x)$ for $x \in \Omega$ (which requires that $\mu$ too has a singularity at $x=1$ ). Use $\lambda\left(\frac{1}{2} a\right)=\lambda(1)=0$, and $\lambda \geqslant 0$. Interpret the result.

EXERCISE 4.3.8: Multiply (4.3.1a) with $x$ and integrate. Show that $k>0$ when $V(x) \geqslant x \mu(x)$ for $x \in \Omega$ while $k \leqslant 0$ when $V(x) \leqslant x \mu(x)$ for $x \in \Omega$. Interpret the result.

\subsection{The inverse problem}

Thus far we have assumed that $V, b$ and $\mu$ are known, but in practice this is hardly ever the case. In fact in cell- and microbiological applications often the main reason for the whole modelling exercise is that we want to use the experimentally obtained (stable) size distribution $\lambda$, and the specific population growth rate $k$ to obtain information about $V, b$ and $\mu$, since it is too difficult (or downright impossible) to determine these functions direct from experimental observations on individual cells. This is called the inverse problem: measurements at the population level are used to get information about physiological processes at the individual level (see also the contributions by Van Straalen and Voorn \& Koch in part B).

The inverse problem comes in many guises, depending on what we may assume to be known. Moreover, there are the practical problems of measurement errors and all other kinds of noise. However, as these notes are first and foremost about modelling we shall start by ignoring these problems, notwithstanding their great practical importance, and concentrate on the identifyability problem per se. Moreover we shall throughout make the simplifying assumption that $\mu=0$ (cell deaths indeed usually are negligible).

Putting $\mu=0$ and integrating once in (4.3.1) we find the identity

$$
V(x)=\frac{1}{\lambda(x)} \int_{a / 2}^{x}[4 b(2 \xi) \lambda(2 \xi)-b(\xi) \lambda(\xi)-k \lambda(\xi)] d \xi
$$

which cell biologists call the equation of Collins \& Richmond. So if $b$ were known we can determine $V$ from $k$ and $\lambda(x)$. Unfortunately $b$ usually is equally unknown. An ingenious experimental way to overcome this difficulty is provided by the observation that $b(x) \lambda(x)$ is proportional to the size distribution $\phi$ of cells which are in the process of division with the constant of proportionality equal to the population growth rate $k$ (in reality division is not instantaneous so that $\phi$ is indeed observable), and $2 b(2 x) \lambda(2 x)$ is proportional to the size distribution $\psi$ of newborn cells (which often are recognizable for a short time after division occurred). So if one can measure either $\phi$ or $\psi$ experimentally one can determine $V$ from (4.4.1).

If $V$ is known but $b$ is not, we can determine $b(x)$ from either

$$
b(x)=-\frac{1}{\lambda(x)} \frac{d(V(x) \lambda(x))}{d x}-k, \quad a \leqslant x<1
$$

sr

$$
b(2 x)=\frac{1}{4 \lambda(2 x)}\left\{\frac{d(V(x) \lambda(x))}{d x}+k\right\}, \quad \frac{1}{2} a \leqslant x<\frac{1}{2} .
$$


(Recall the assumption $a \geqslant \frac{1}{2}$.) The requirement that these two formulae should yield a similar appearance of $b$ can be $\lambda$ in the form as for an assumed form of $V$. Unfortunately the fact that measurements usually yield information about $\lambda$ in the form of a histogram somewhat spoils the easy elegance of these formulae.
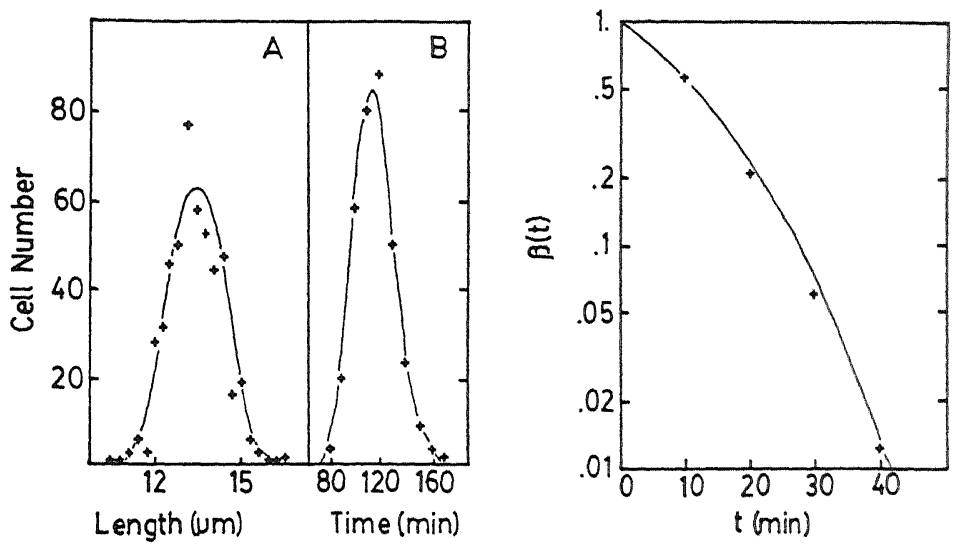

Fig. 4.4.1 Probability density of division size $(A)$ and generation time $(B)$ and probability $\beta$ that two sister cells differ in generation time by $t(C)$, for an exponentially growing population of fission yeast (Schizosaccharomyces pombe). Data from Miyata et al (1978). The continuous curves are predictions from the model with $V(x)=k x$ and $b(x)=\hat{b}(x-a)^{2} /(1-x)$ for $x>a, a=0.6$ and $\hat{b} / k=100$. From TYSON \& DIEKMANN (1986).

When data are few and noisy, as they almost invariably are, the only way to proceed is to make plausible guesses for the functional forms of $b$ and $V$, still depending on a limited number of parameters. Subsequently the values of the parameters are estimated by juggling them around to get as good a match as possible between $\lambda$ calculated from the explicit expression (4.3.4) and the experimentally observed size distribution. The closeness of the fit obtained provides a first check of the model. However, the really convincing checks are provided by the ability of the model to predict other experimentally observable quantities. An example is provided by the work of TYson \& DiekMANN (1986), who, using data on fission yeast reported by MiYata, MrYATA \& ITo (1978) fitted the "sloppy size control" model of this section in the way indicated, and subsequently found remarkably good agreement between observed and predicted generation time distributions (see fig. 4.4.1).

We conclude our too brief sketch with stressing once more that the inverse problem has many variants and is frought with many subtle difficulties, which are worthy of a great deal of attention. But this is all outside the scope of this chapter. The paper by Voorn \& Koch in part B deals with the moment relations between the various distributions involved, using (a generalized version of the Collins-Richmond equation as its main tool. Some interesting early references which also contain concrete applications, are Bell \& ANDerson (1967) and Anderson, Bell, Petersen \& TOBEY (1969)

\subsection{Limits to growth}

In the previous sections we found that the number of cells will grow exponentially when $k>0$. In reality this will be prevented by exhaustion of space, nutrients etc. In our model conception the causal chain leading to a stagnation of population growth starts with a stagnation in individual growth which in turn slows down the rate of cell fission, and thereby the increase in cell numbers. In this section we shall assume that it is the nutrients which are in short supply. In that case the obvious way to model the feedback loop between population size and nutrient availability is to introduce an additional finite dimensional variable $R$ representing the availability of the various necessary nutrients and to specify (i) how the individual growth rate depends on $R$, (ii) how nutrient consumption depends on both $R$ and cell size, and (iii) what other contributions there are to the change in $R$. Since in this set up the growth rate depends implicitly on time the distinction made in subsection 4.1 between various conceptions about the detailed probabilistic nature of the fission process becomes very relevant. In this section we shall opt for the stochastically predetermined division size, or stochastic threshold, assumption, mainly since this choice allows us to exploit the linear machinery developed previously (and more fully in chapter II) to analyse an inherently nonlinear situation. We refer to chapter 
VI for a discussion of some other approaches.

EXERCISE 4.5.1: Discuss ways to distinguish experimentally between the two possible assumptions about the division process from subsection 4.1 by manipulating the food availability. Which of the two possibilities has a greater plausibility in your opinion?

For definiteness we shall assume that the food dynamics is that of a chemostat. This is a vessel into which a nutrient broth is pumped at a constant rate, while excess medium is removed at the same rate so that the total living volume remains the same. (see figure 4.5.1.) The vessel is continually stirred to keep the cells in suspension and to guarantee spatial homogeneity of the culture conditions. We shall assume moreover that all nutrients except one socalled limiting nutrient (also called limiting substate) are available in excess, making $R$ effectively one dimensional.

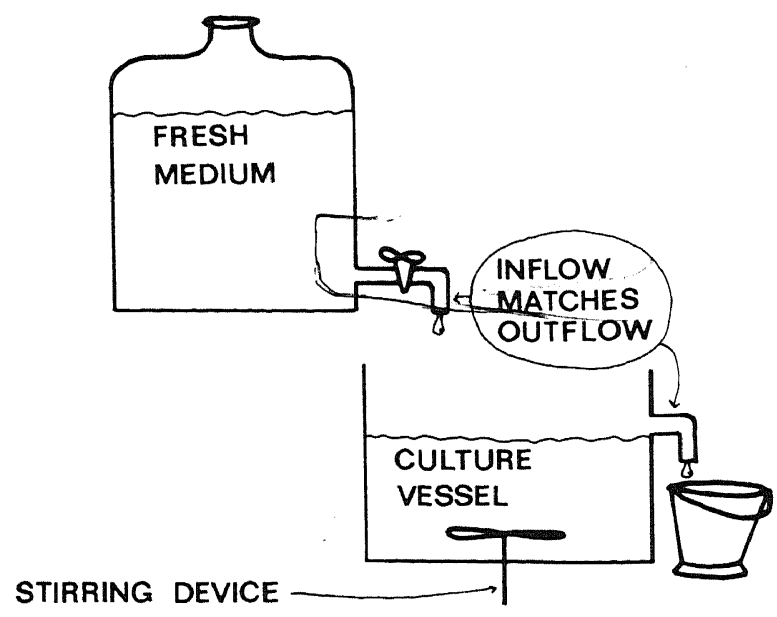

Fig. 4.5.1. Schematic representation of a chemostat

Moreover we assume that the growth rate factorizes in the sense that (with some abuse of notation)

$$
V=\beta(R) V(x)
$$

In Diekmann, LAUWerier, AIDENBerg \& METz (1984) this is called the structural nutrient hypothesis, since it seems a reasonable assumption when the growth rate is limited by the uptake of nutrients like nitrate or phosphate which are all transformed into cell biomass, as opposed to energy which also is consumed in basal metabolism (compare the Daphnia model of the previous section).

REMARK 4.5.2: The assumption commonly made in the microbiological literature is that $\beta$ is the Monod (or Michaelis-Menten) function

$$
\beta(R)=\frac{k_{1} R}{1+k_{2} R}
$$

(compare section 3.1), which is approximately proportional to $R$ for small $R$ and saturates for large $R$.

In combination with the stochastic threshold assumption (4.5.1) gives us for the division rate

$$
b(R, x)=\beta(R) V(x) \delta(x),
$$

where

$$
\delta(x)=\frac{\phi_{b}(x)}{1-\int_{a}^{x} \phi_{b}(\xi) d \xi}
$$

Finally, keeping to the structural nutrient interpretation also forces us to assume that the consumption of limiting 
nutrient by the cell population as a whole is just proportional to the total biomass accretion of the cell population $\int_{a / 2}^{1} \beta(R) V(x) n(t, x) d x$. If we also assume that cell death can be neglected, so that the only "death" term is due to washout of cells in the
effluent medium we get

$$
\begin{aligned}
& \left.\frac{\partial}{\partial t} n(t, x)\right)=\beta(R(t))\left\{-\frac{\partial}{\partial x}(V(x) n(t, x))-V(x) \delta(x) n(t, x)+4 V(2 x) \delta(2 x) n(t, 2 x)\right\}-D n(t, x) \\
& \frac{d R}{d t}=D\left(R_{i}-R(t)\right)-\alpha \beta(R(t)) \int_{a / 2}^{1} V(\xi) n(t, \xi) d \xi
\end{aligned}
$$

where

$$
\begin{aligned}
& D \equiv \text { dilution rate } \equiv \frac{\text { flow rate of medium }}{\text { volume of culture vessel }} \\
& R_{i} \equiv \text { concentration of limiting substrate in the fresh medium } \\
& \alpha \equiv \text { conversion factor relating biomass units substrate units }
\end{aligned}
$$

EXERCISE 4.5.3: Check that total biomass $W(t)=\int_{a / 2}^{1} x n(t, x) d x$ satisfies

$$
\frac{d W(t)}{d t}=\beta(R(t)) \int_{a / 2}^{1} V(\xi) n(t, \xi) d \xi-D W
$$

and that $Z(t)=\alpha W(t)+R(t)$ satisfies

$$
\frac{d Z}{d t}=D\left(R_{i}-Z(t)\right)
$$

and that consequently $Z(t) \rightarrow R_{i}$ for $t \rightarrow \infty$. Also argue that this result is immediately obvious from the (chemical) interpretation of $Z$.

From the way equation (4.5.5) is written it appears that the change in the size distribution is made up of two components: (i) the usual growth cum division, and (ii) washout. The nutrient availability only influences the first process by changing its overall speed. As the washout is not size selective it only influences population numbers but not the relative frequencies of the cell sizes. In other words, the process by which the size distribution equilibrated in the linear case is still there except that it now proceeds at a variable speed. This idea can be captured in the following theorem which is proved in chapter II:

THEOREM 4.5.4: Suppose that $V(2 x) \neq 2 V(x)$ for some $x \in\left(\frac{a}{2}, \frac{1}{2}\right]$. Then for large $t$ the solution to (4.5.4) behaves like

$$
n(t, x)=\rho(t)\{\lambda(x)+o(1)\}
$$

where $\rho$ satisfies

$$
\frac{d \rho}{d t}=(k \beta(R)-D) \rho
$$

and where $\lambda$ and $k$ are defined by (4.3.4) and (4.3.3) resp., with $\mu(x)=0$ and $b(x)=V(x) \delta(x)$.

Substituting (4.5.9) in (4.5.6) gives, neglecting the o(1) term,

$$
\frac{d R}{d t}=D\left(R_{i}-R\right)-\alpha_{0} \beta(R) \rho
$$

with

$$
\alpha_{0}=\alpha \int_{a / 2}^{1} V(\xi) \lambda(\xi) d \xi .
$$

(4.5.10) and (4.5.11) together govern the asymptotic behaviour of $\rho$ and $R$, and by (4.5.9) also the asymptotic behaviour of the solution to (4.5.5) and (4.5.6). 
Equations (4.5.10) and (4.5.11) are just the traditional chemostat equations of unstructured population dynamics, the asymptotic behaviour of which is well known (see the exercises below), implying that here at least we have a structured population model which can be solved in toto; but at the cost of some rather special, even if not unreasonable, assumptions.

An indirect check of our special assumptions is provided by the prediction that the equilibrium size distribution (which can easily be observed noninvasively in the effluent medium) should be independent of the chemostat parameters $D$ and $R_{i}$. One set of possibly relevant data can be found in Williams (1971). These certainly appear to be at variance with our prediction (but unfortunately the experimental conditions are indicated rather loosely, so that this cannot be considered a definite check even in this special case). We would be very grateful for any future data of this type. Only by considering a large number of special cases it is possible to evaluate the range of our approach.

Even without any experimental evidence it can be seen that there should be a wide range of cases in which our simplifying assumptions cannot possibly hold good. Therefore there is every reason to study other special cases in which either (i) the function $V=V(R, x)$ is not the product of two factors $\beta(R)$ and $V(x)$, or (ii) $b$ is not a product of $V(R, x)$ and some function $\delta(x)$ indepent of $R$. Unfortunately such cases are essentially more complicated since it will no longer be possible to reduce the problem to a linear one through a simple time scaling trick. We are inevitably led to enter the domain of infinite dimensional nonlinear dynamical systems; but this is postponed to chapter VI.

EXERCISE 4.5.5: Show that

$$
\int_{a / 2}^{1} V(\xi) \lambda(\xi) d \xi=k \int_{a / 2}^{1} \xi \lambda(\xi) d \xi .
$$

What is the interpretation of this relation? Hint: Use (4.3.1) with $\mu=0$.

EXERCISE 4.5.6: Let $\beta$ be a strictly increasing function with $\beta(0)=0$ and $\beta(\infty)<\infty$. We denote by $\beta^{-1}$ the inverse function of $\beta$. Show that the system (4.5.10) and (4.5.11) has equilibrium points $\left(0, R_{i}\right)$ and $(\hat{\rho}, \hat{R})$ where

$$
\hat{\rho}=\frac{k}{\alpha_{0}}\left(R_{i}-\beta^{-1}(D / k)\right) \quad \hat{R}=\beta^{-1}(D / k)
$$

provided that $D / k<\beta\left(R_{i}\right)$. If $D / k \geqslant \beta\left(R_{i}\right)$ only the trivial equilibrium remains. Interpret this difference between the two cases in biological terms.

EXERCISE 4.5.7: Use the result of exercise (4.5.3) together with theorem 4.5.4 to show that

$$
R(t)+\frac{\alpha_{0}}{k} \rho(t) \rightarrow R_{i} \text { for } t \rightarrow \infty .
$$

Conclude that the asymptotic behaviour of $\rho$ is described by the scalar differential equation

$$
\frac{d \rho}{d t}=\left(k \beta\left(R_{i}-\frac{\alpha_{0}}{k} \rho\right)-D\right) \rho,
$$

and from this that all solutions converge towards $\rho=0$ when $\beta\left(R_{i}\right) \leqslant D / k$ and to $\rho=\hat{\rho}$ when $\beta\left(R_{i}\right)>D / k$.

EXERCISE 4.5.8: Discuss how $\beta$ can be estimated by observing the composition of the effluent medium while (slowly) changing the flow rate. Hint: How would you determine $\alpha_{0}, k$ ?

EXERCISE 4.5.9: Assume that two species of microorganisms satisfying all the assumptions of this subsection and both dependent on the same limiting substrate are grown together in a chemostat. Analyse the outcome of the competition on the assumption that $\beta_{1}$ and $\beta_{2}$ both strictly increase with $R$. Hint: analyse the ordinary differential equations describing the large time behaviour, using the asymptotic conservation condition for total nutrient concentration to reduce the problem to a two-dimensional one.

EXERCISE 4.5.10: Consider a microorganism which satisfies all the assumptions of this subsection except for the structural nutrient hypothesis. Assume that $\partial V(x, R) / \partial R>0$. Analyse the existence and uniqueness of any nontrivial equilibrium. Hint: Follow the pattern laid out in the Daphnia story of the previous section.

\subsection{Summary}

Following Bell \& ANDERSON (1967), Fredrickson, RAMKRishna \& TSUCHIYA (1967) and Sinko \& Streifer (1967) 
we have derived a balance law for the size distribution of unicellular organisms reproducing by fission. Basic ingredients for this equation were a deterministic (mechanistic, physiological) conception of individual growth and a statistical description of fission and death. In doing so we hit upon some unexpected, mechanistic ambiguities inherent in the statistical description which only become overt when individual growth is under environmental control: is the probability of division related to the crossing of a certain size range or does it depend on the available time?

By means of the semigroup theory developed in chapter II it can be shown that generally under constant environmental conditions the population will grow exponentially with rate parameter $k$ while the size distribution stabilizes, the exception being provided by the case of individual growth rates satisfying $V(2 x)=2 V(x)$ (which includes the biologically non-trivial case of exponential $i$-growth) and equal division. We have moreover given an equation from which $k$ can be calculated, as well as an explicit expression for the asymptotic size distribution, and we have briefly indicated how these results may help to solve the inverse problem of determining the individual characteristics from data on the population growth rate and size distribution.

Finally we discussed substrate limited growth in a chemostat for the special case that (i) the probability of fission depends on the size range crossed, and (ii) the individual growth rate can be written as a product of a size dependent and a nutrient dependent term (the structural nutrient hypothesis). These two assumptions were found to guarantee that the size distribution converges to a stable distribution which is independent of the chemostat parameters, and that total population size and substrate concentration asymptotically satisfy the usual chemostat equations of unstructured population dynamics.

We ended with a plea for the study of nonlinear structured cell population models under less restricted conditions.

\section{On semigroups and generators}

Underlying the three examples we have just treated is one abstract, mathematical pattern. In this final section we shall bring this pattern out in the open, both for its own intrinsic interest and as introduction to the ensuing mathematical chapters. For here we have one of those pleasing cases where in fact the biological imagery and the mathematical toolkit have converged to a considerable extent.

The basic ingredient in our approach was the concept of population state, in the guise of a density function

$$
x \mapsto n(t, x) .
$$

Here "state" is interpreted as "an amount of information about the population sufficient to completely fix the future development of that population". In other words, if we know at $t_{0}$ the function $n\left(t_{0},{ }^{\circ}\right)$ and the time course of the environment, then at least in principle we can calculate $n(t, \cdot)$ for all $t>t_{0}$.

In all the cases of variable environments we have dealt with, the environment itself was subject to dynamical equations, just like the population was, with the state of the population as the only input, making the total, coupled system autonomous, i.e. independent of outside influences (mathematically this means that all our equations are invariant under a translation of the time axis). Below we shall concentrate on autonomous systems only. (A rough, heuristic sketch of the framework needed for dealing with general non-autonomous systems is given in chapter III.) Moreover we shall assume for definiteness that the only changes are in the population as this simplifies the notation, and, less harmlesly, we shall restrict ourselves mainly to linear systems, i.e. systems in which there also is no direct interaction between the individuals of our population.

For an autonomous system an initial condition

$$
n(0, x)=n_{0}(x),
$$

(with $n_{0}$ assumed to be known) completely and uniquely determines $n(t, \cdot)$ for all $t \geqslant 0$. Sometimes we write $n\left(t, x, n_{0}\right)$ to emphasize this dependence on the initial condition. Another way of expressing that $n_{0}$ for any given $t$ completely determines $n(t, \cdot)$ is to write

$$
n\left(t, x, n_{0}\right)=\left(S(t) n_{0}\right)(x)
$$

where for each $t \geqslant 0$ the symbol $S(t)$ stands for an operator, which maps functions of $x$ into functions of $x$. (See fig. 5.1).

The definition immediately implies

$$
\begin{aligned}
& S(0)=I \\
& S(t) S(\tau)=S(t+\tau) \quad t, \tau \geqslant 0
\end{aligned}
$$




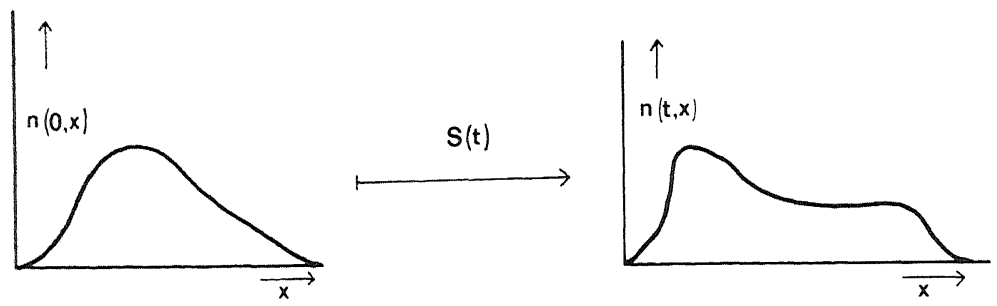

Fig. 5.1. The action of the next state operator $S$ on the population state

One calls $(5.3 \mathrm{~b})$ the semigroup property, where the prefix "semi" reflects the restriction to positive $t$.

EXERCISE 5.1: Use uniqueness and translation invariance to verify (5.3b). Hint: Refer to figure 5.2.

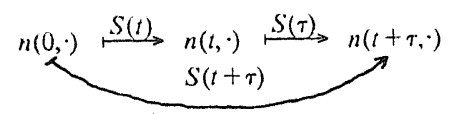

Fig. 5.2. The causal chain connecting the population state at $t+\tau$ to the initial state through the state at the intermediate time $t$.

There exists an extensive mathematical theory of (one-parameter) semigroups of linear operators (Pazy, 1983a). Here one considers a family $\{S(t)\}_{t \geqslant 0}$ of linear operators with properties (5.3a, b) and in addition some continuity properties. The interesting thing is that one can arrive at such semigroups direct from the biological interpretation. This should motivate a study of the corresponding mathematical theory. Chapter II gives an introduction especially geared towards applications in structured population models.

From a fundamental point of view the operators $S(t)$ are precisely the objects of one's inquiry. But in practice they are hard to obtain direct in explicit form. Indeed, how can we express them in terms of for example the functions $V, b$ and $\mu$ characterizing the behaviour of individual cells, as introduced in the previous section? In finite time intervals the intrinsic entanglement of the effects of growth, births and deaths almost invariably complicates the bookkeeping to an unmanageable extent. The solution to this problem is as old as the invention of differential calculus: in small time intervals there will hardly be any "compound" events, and in the limit they can be neglected altogether. In other words, in infinitesimal time intervals the contributions of the different processes to the change in the population state are uncoupled and therefore easily calculated. So instead of directly specifying $S(t)$ we derive differential equations.

Abstractly we can write

$$
\frac{d n}{d t}=A n \quad n(0)=n_{0}
$$

where the operator $A$ (acting on functions of $x$ ) is defined by

$$
A m=\lim _{t \downarrow 0} \frac{1}{t}(S(t) m-m)
$$

for those functions $m$ for which this limit exists indeed. One calls $A$ the infinitesimal generator of the semigroup $S(t)$. For example in the last example (the cell population) $A$ is defined by

$$
(A m)(x)=-\frac{d V(x) m(x)}{d x}-b(x) m(x)+4 b(2 x) m(2 x)-\mu(x) m(x),
$$

$m:\left[\frac{1}{2} a, 1\right) \rightarrow \mathbb{R}$ a representative $i$-state distribution, together with the condition that $m$ is such that the expression on the right hand side makes sense and

$$
m\left(\frac{1}{2} a\right)=0
$$

(Operators, like all maps, are defined by telling what their domain is and by giving a recipe to calculate the result of 
their action).

Equation (5.4) with (5.6) is just an abstract reexpression of our old equation (4.2.1). The difference is that (5.4) is an (abstract) ordinary differential equation for the function $n$ of one variable $t$ which assumes values in a space of functions of $x$ (so that we can write $n(t)(x)$ ), whereas (4.2.1) is a (functional) partial differential equation. It should be clear that these are just two ways of looking at the same object. However the first way certainly is the more protitable one.

In chapter II we shall sketch how for linear structured population models one can construct the unique solution to (5.4) by means of a so-called generation expansion, i.e. a series representation in which the subsequent terms are just the $i$-state densities of the individuals in the subsequent biological generations (in the predation example we shall consider a jump in satiation as a combined death-birth event). Unfortunately no such general technique is available in the nonlinear case. In chapter VI some examples are given how one may proceed to prove existence and uniqueness of solutions in some special cases.

The generation expansion construction is quite useful for calculating $S(t)$ for small $t$, but clearly it is unsuited to the calculation of the large time behaviour. For the latter purpose we have to take recourse to spectral theory, much in the same way as the asymptotic behaviour of the solution of the ordinary differential equation $d X / d t=B X, X(t) \in \mathbb{R}^{n}$ and $B$ and $n \times n$ matrix, can be determined with the aid of the eigenvalues and eigenvectors of $B$. The extension of this theory from the finite to the infinite dimensional case is beset with subtle and mathematically interesting difficulties, keeping it an area of ever active mathematical research. Luckily in the case of structured population models there is a helpful constraint deriving direct from the interpretation: population densities are nonnegative. So the semigroup $S(t)$ should leave the so-called cone of nonnegative functions invariant. By exploting this positivity property it can often be shown (in chapters II and V it will be told how) that the eigenvalue equation

$$
A \nu=\lambda \nu
$$

allows precisely one nonnegative eigenfunction $\nu$ and a corresponding real eigenvalue $\lambda$ (compare equation (4.3.1), equations (3.3.2), (3.3.5) and (3.3.6), and equation (2.6.1a); in the latter equation the conservation of total predator number guarantees that $\lambda=0$ ). Moreover the space of possible population states can be decomposed into two components, a one-dimensional space spanned by $\nu$ and a "remainder" component, such that

$$
n(t, x)=c\left(n_{0}\right) e^{\lambda t} \nu(x)+R\left(t, x, n_{0}\right)
$$

$\left(c\left(n_{0}\right)\right.$ a real number), where the remainder $R$ is such that

$$
e^{-\lambda t} R(t, x) \rightarrow 0
$$

in some appropriate sense.

The eigenfunction $\nu$ is the stable $i$-state distribution which kept recurring in our discussions, and the corresponding eigenvalue $\lambda$ is the intrinsic rate of natural increase. In the first example of this chapter apparently $\lambda=0$, and in the other two examples it was possible to derive a relatively simple characteristic equation from which $\lambda$ can easily be cal-

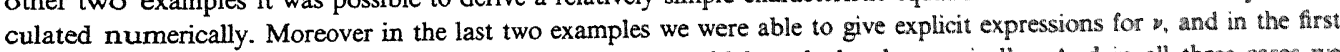
example we had available an easy recipe from which $\nu$ could be calculated numerically. And in all three cases we could reasonably argue that the essential biological information provided by the model was contained precisely in that real number $\lambda$ and that eigenfunction $\nu$.

EXERCISE 5.1: Give another explanation, phrased in terms of eigenvalues and characteristic equations, why the solution to (2.6.1) automatically satisfies the side condition (2.5.5b). 